Florida International University FIU Digital Commons

6-12-2014

\title{
Analysis of Kepler Active Galactic Nuclei Using A Revised Kirk, Rieger, Mastichiadis (1998) Model
}

Sarah M. Dhalla

Florida International University, sarahdhalla@gmail.com

DOI: $10.25148 /$ etd.FI14071133

Follow this and additional works at: https://digitalcommons.fiu.edu/etd

\section{Recommended Citation}

Dhalla, Sarah M., "Analysis of Kepler Active Galactic Nuclei Using A Revised Kirk, Rieger, Mastichiadis (1998) Model" (2014). FIU Electronic Theses and Dissertations. 1521.

https://digitalcommons.fiu.edu/etd/1521 


\title{
FLORIDA INTERNATIONAL UNIVERSITY
}

\author{
Miami, Florida
}

\section{ANALYSIS OF KEPLER ACTIVE GALACTIC NUCLEI USING A REVISED KIRK, RIEGER, MASTICHIADIS (1998) JET MODEL}

\author{
A thesis submitted in partial fulfillment of the \\ requirements for the degree of \\ MASTER OF SCIENCE \\ in \\ PHYSICS \\ by
}

Sarah Mohammed Dhalla

2014 
To: Interim Dean Michael R. Heithaus

College of Arts and Sciences

This thesis, written by Sarah Mohammed Dhalla, and entitled Analysis of Kepler Active Galactic Nuclei Using a Revised Kirk, Rieger, \& Mastichiadis (1998) Jet Model, having been approved in respect to style and intellectual content, is referred to you for judgment.

We have read this thesis and recommend that it be approved.

Caroline Simpson

Walter Van Hamme

James R. Webb, Major Professor

Date of Defense: June 12, 2014

The thesis of Sarah Mohammed Dhalla is approved.

Interim Dean Michael R. Heithaus

College of Arts and Sciences

Dean Lakshmi N. Reddi

University Graduate School

Florida International University, 2014 
C Copyright 2014 by Sarah Mohammed Dhalla

All rights reserved. 


\section{DEDICATION}

To my family 


\section{ACKNOWLEDGMENTS}

A big thanks to my advisor and my committee members for helping me through this process.

This paper includes data collected by the Kepler mission. Funding for the Kepler mission is provided by the NASA Science Mission directorate. 


\section{ABSTRACT OF THE THESIS \\ ANALYSIS OF KEPLER ACTIVE GALACTIC NUCLEI USING A REVISED KIRK, RIEGER,\& MASTICHIADIS (1988) JET MODEL}

by

Sarah Mohammed Dhalla

Florida International University, 2014

Miami, Florida

Professor James R. Webb, Major Professor

Active Galactic Nuclei (AGN) are cores of distant protogalaxies, with a supermassive blackhole at the center surrounded by an accretion disk, and bipolar jets. Blazars, a subset of AGN, have their jets aligned with our line of sight. Emission from blazars is highly variable on all timescales and frequencies. Microvariabilityrefers to rapid continuum variations that arise within the jet. Bhatta et al. (2013) suggest a modified Kirk, Rieger, \& Mastichiadis (1998) model (KRM) to explain microvariability. The KRM model assumes that when shock waves passes though the jet, each turbulent cell encountered produces a pulse of emission characterized by cell size, local density enhancement, and magnetic field strength. NASA's Kepler has monitored optical emission from four AGN. We use the modified KRM model to analyze micro-variations in these Kepler data. The distribution of cell sizes computed from these data is consistent with the distribution expected from a turbulent plasma. 


\section{TABLE OF CONTENTS}

CHAPTER

PAGE

1 Introduction 1

1.1 Active Galactic Nuclei (AGN) $\ldots \ldots \ldots \ldots \ldots \ldots$

1.2 History of AGN . . . . . . . . . . . . . . . . 2

1.3 AGN Classification . . . . . . . . . . . . . . . . . . 3

1.4 Blazar Variability . . . . . . . . . . . . . . . . . . . . 5

2 Modeling Microvariability $2 \ldots$

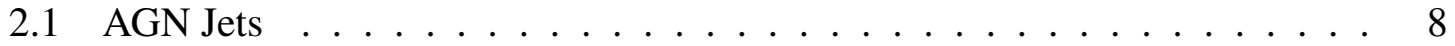

2.2 Model . . . . . . . . . . . . . . . . . . . . . . 9

2.3 Revised KRM Model . . . . . . . . . . . . . . . . . . . . . . 10

2.4 Previous Data $\ldots \ldots \ldots \ldots \ldots \ldots$

3 Multi-color Study of 0716+71 19

4 The Kepler Mission 23

$4.1 \quad$ Kepler Space Telescope . . . . . . . . . . . . . . . . . . . . . . 23

4.2 Kepler Data Pipeline . . . . . . . . . . . . . . . . . . 26

5 Data Analysis 29

$5.1 \quad$ Pulse Fitting . . . . . . . . . . . . . . . . . . . . . . . . 29

$5.1 .1 \quad \mathrm{Zw} 229-15 \ldots \ldots \ldots \ldots$

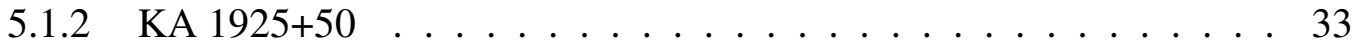

$5.1 .3 \quad$ KA $1858+48 \ldots \ldots \ldots \ldots \ldots \ldots \ldots$

5.1 .4 KA $1904+37 \ldots \ldots \ldots \ldots \ldots \ldots \ldots$

6 Conclusion and Future Work 44

$\begin{array}{ll}\text { List of References } & 41\end{array}$

$\begin{array}{ll}\text { Appendices } & 43\end{array}$ 


\section{LIST OF TABLES}

TABLE

PAGE

1 WEBT Campaign Data ..................... 16

2 Kepler AGN Source Information . . . . . . . . . . . . . . . . 27

3 Pulse Fitting Data . . . . . . . . . . . . . . . . . . . . . . . 29

4 Zw 229-15 Pulse Fitting Data- Cadence $6 \ldots \ldots$. . . . . . . . . . . . 50

$5 \quad \mathrm{Zw}$ 229-15 Pulse Fitting Data- Cadence $7 \ldots \ldots$. . . . . . . . 51

6 KA 1858+48 Pulse Fitting Data- Cadence $6 \ldots \ldots$. . . . . . . . . 53

7 KA $1858+48$ Pulse Fitting Data- Cadence $7 \ldots \ldots 5$

8 KA $1925+50$ Pulse Fitting Data- Cadence $6 \ldots \ldots$. . . . . . . . . 57

9 KA $1925+50$ Pulse Fitting Data- Cadence $7 \ldots \ldots$. . . . . . . . . . 60

10 KA 1904+37 Pulse Fitting Data- Cadence $7 \ldots \ldots 2$ 


\section{LIST OF FIGURES}

FIGURE

PAGE

1 A model of AGN by Urry and Padovani . . . . . . . . . . . . . . 2

2 Classifications of AGN depending on their observational angle. . . . . . . . 4

3 A model of a turbulent jet . . . . . . . . . . . . . . . 9

4 Revised KRM model . . . . . . . . . . . . . . . . . . . . . . 10

5 Pulse shapes for two different frequencies . . . . . . . . . . . . 14

6 Clockwise hysteresis loop . . . . . . . . . . . . . 15

7 Counter-Clockwise hysteresis loop . . . . . . . . . . . 15

8 Results of the 2009 WEBT Campaign _ . . . . . . . . . . . . . . . . 17

$9 \quad \mathrm{~V}$ band light curve of $0716+71$ fitted with 7 pulses . . . . . . . . . . 18

10 I band light curve of $0716+71$, fitted with 8 pulses . . . . . . . . . . . . 19

11 Spectral index vs. V band flux ................. 20

$12 \mathrm{~V}$ (green) and I (red) band pulse shape . . . . . . . . . . . . . 20

13 Kepler's field of view, centered in the constellation Lyra and Cygnus . . . . 22

14 Kepler spacecraft ....................... 23

15 Sample light curve of $\mathrm{Zw} 229-15 \ldots \ldots \ldots \ldots$

16 SAP (red) and PDC SAP (blue) light curves . . . . . . . . . . . 25

17 Shape of the Kepler pulse $($ frequency $=575 \mathrm{~nm}) \quad \ldots \ldots$. . . . . . . 27

$18 \mathrm{Zw} 229-15$ cadence 6 fitted light curve . . . . . . . . . . . . . 32

19 Zw 229-15 cadence 7 fitted light curve . . . . . . . . . . . . . . 32

20 Zw 229-15 cadence 6 and 7 frequency distribution of cell size . . . . . . . 33

21 KA $1925+50$ cadence 6 fitted light curve . . . . . . . . . . . . . . 34

22 KA $1925+50$ cadence 7 fitted light curve . . . . . . . . . . . . . . 34 
23 KA $1925+50$ cadence 6 and 7 frequency distribution of cell size . . . . . 35

24 KA $1858+48$ cadence 6 fitted light curve . . . . . . . . . . . . . . . 36

25 KA $1858+48$ cadence 7 fitted light curve . . . . . . . . . . . . 36

26 KA 1858+48 cadence 6 and 7 frequency distribution of cell size . . . . . 37

27 KA $1904+37$ cadence 7 fitted light curve . . . . . . . . . . . . . . . 37

28 KA 1904+37 cadence 7 frequency distribution of cell size . . . . . . . . 38 


\section{Introduction}

\subsection{Active Galactic Nuclei (AGN)}

Active Galactic Nuclei (AGN) are the extremely luminous and unresolved cores of distant galaxies. Current AGN models propose a Super Massive Black Hole (SMBH) as

the central engine surrounded by an accretion disk. This accretion disk is thought to supply the SMBH by the injection of matter and magnetic fields. Some AGN are strong sources of radio emission (radio-loud quasars). These AGN generally have bipolar jet outflows that extend from the central core of the SMBH and move outwards at relativistic speeds $(0.95 \mathrm{c}$ to $0.99 \mathrm{c})$ to a few kiloparsecs $(\mathrm{kpc})$ from the central core. Figure 1 illustrates the AGN model from Urry \& Padovani (1995). Compared to a typical galaxy, AGN have $10^{2}$ to $10^{4}$ times the power output with luminosities ranging from a reported $10^{42} \mathrm{erg} / \mathrm{s}$ to $10^{48}$ erg/s. However, because some of the AGN cores are obscured by dust and others may be affected by relativistic beaming, these cores may be much more luminous than reported. When viewed in the optical, AGN cores appear as unresolved point sources with angular sizes of much less than 1 arcsec $\left({ }^{\prime \prime}\right)$.

A key property of AGN is flux variability. Flux variability is observed in all frequencies and on all timescales. Observations across the electromagnetic spectrum show that the amplitude of these variations is dependent on frequency. For example, high amplitude optical variability is often associated with a compact radio structure and strong high energy $\gamma$-ray emission. The emission is also linearly polarized because of synchrotron radiation, thus distinguishing the AGN from stars and galaxies.

Many AGN also have spectra that are characterized by broad optical emission lines, which is unlike any typical galaxy. Galaxies generally show a thermal spectrum with stellar absorption lines. The QSO broad emission lines are thought to originate from thick clouds that are near the central region and are known as the Broad-Line emission Region 


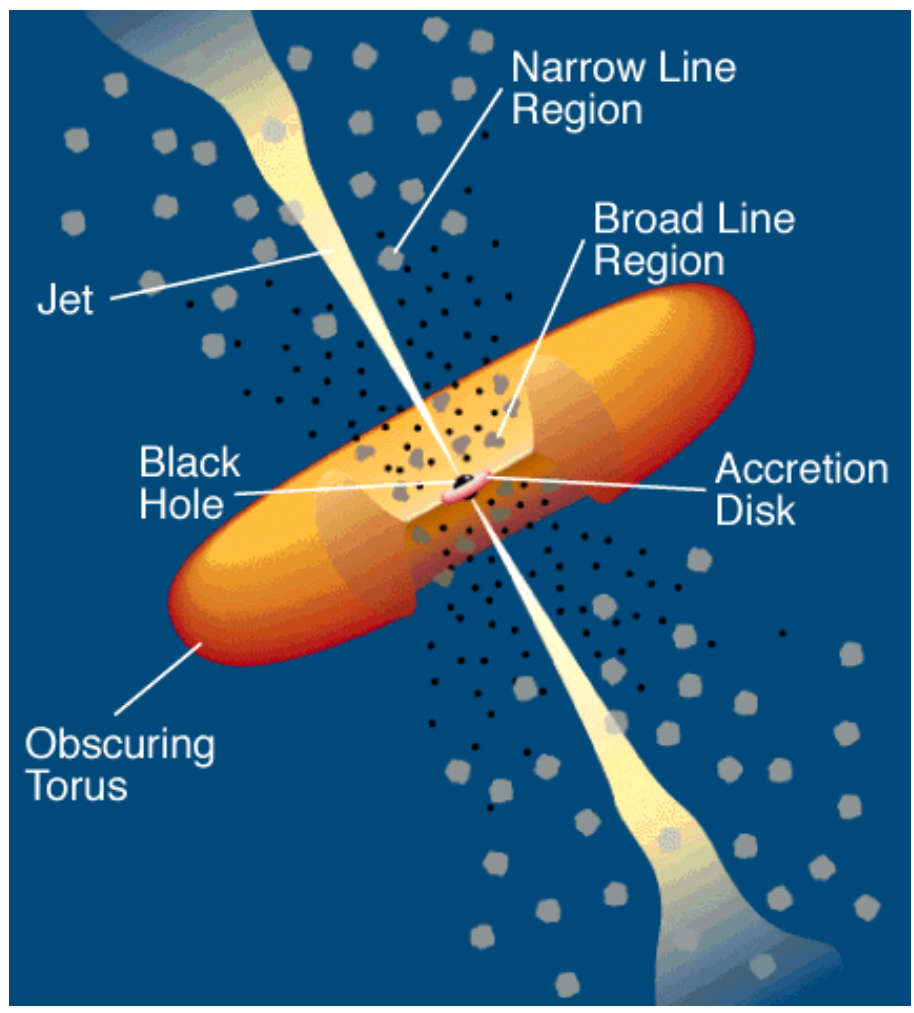

Fig. 1.- AGN model by Urry and Padovani (1995) illustrating the components of AGN which consist of the central engine, accretion disk and bipolar jets. ${ }^{1}$

(BLR). Narrow lines in the AGN spectra emanate from a thick cloud that encloses the central broad line region. The BLR region consists of relatively low density clouds several $\mathrm{kpc}$ away from the central engine. In fact it is because of their unique spectra that AGN were actually first identified as new objects in the universe.

\subsection{History of AGN}

In 1960 Allan Sandage obtained a photograph of radio source 3C 48 which showed a faint nebulosity surrounding a stellar object. The spectrum showed broad emission lines at unfamiliar wavelengths and photometry showed that the stellar-like emission was also

\footnotetext{
${ }^{1}$ http://heasarc.gsfc.nasa.gov/docs/cgro/images/epo/gallery/agns/index.html
} 
variable with an excess of ultraviolet emission. Several other objects similar to $3 \mathrm{C} 48$ were found and named quasi-stellar radio sources (QSRS/QSO) or quasars. Allan et al. (1962) found that $3 \mathrm{C} 273$ consisted of two components: the underlying nucleus and a bright radio jet emanating from a stellar, point-like source. Maarten Schmidt in 1963 was pondering the optical spectrum of quasar 3C 273 and found that the emission lines at unfamiliar wavelengths were actually the Balmer series of hydrogen with a redshift of $\mathrm{z}=0.158$ (Schmidt 1963). He conjectured that the extreme redshift was the result of cosmological expansion (receding at a rate of 47,000 km/s). This made 3C 273 an extremely distant object.

During the same time, Cuno Hoffmeister discovered and observed a variable star named BL Lacertae (BL Lac). Later, MacLeod and Andrew (1968) associated a radio source with BL Lac. BL Lacertae's flat spectrum had no emission or absorption lines. Later, in the 1970s, BL Lac demonstrated the same radio characteristics as other AGN and because the spectral and radio observations showed that BL Lac behaved similarly to many AGN, but lacked broad emission lines, it was classified as separate type of AGN. These different classifications are described below.

\subsection{AGN Classification}

Active galactic nuclei are classified by many properties that they exhibit. Since there is no one universal classification system, some AGN may fall into two or more categories based on their observed properties. In general, they are classified on the basis of their radio, optical or $\gamma$-ray emission. An attempt to reconcile this with a physical picture led to the model where you see different AGN depending on the observed angle to the line of sight (see Figure 2).

In the viewing angle classification system, Seyfert galaxies are a class of AGN that are observed at an angle of $0^{\circ}$ to $30^{\circ}$ with respect to the disk. They were originally divided 


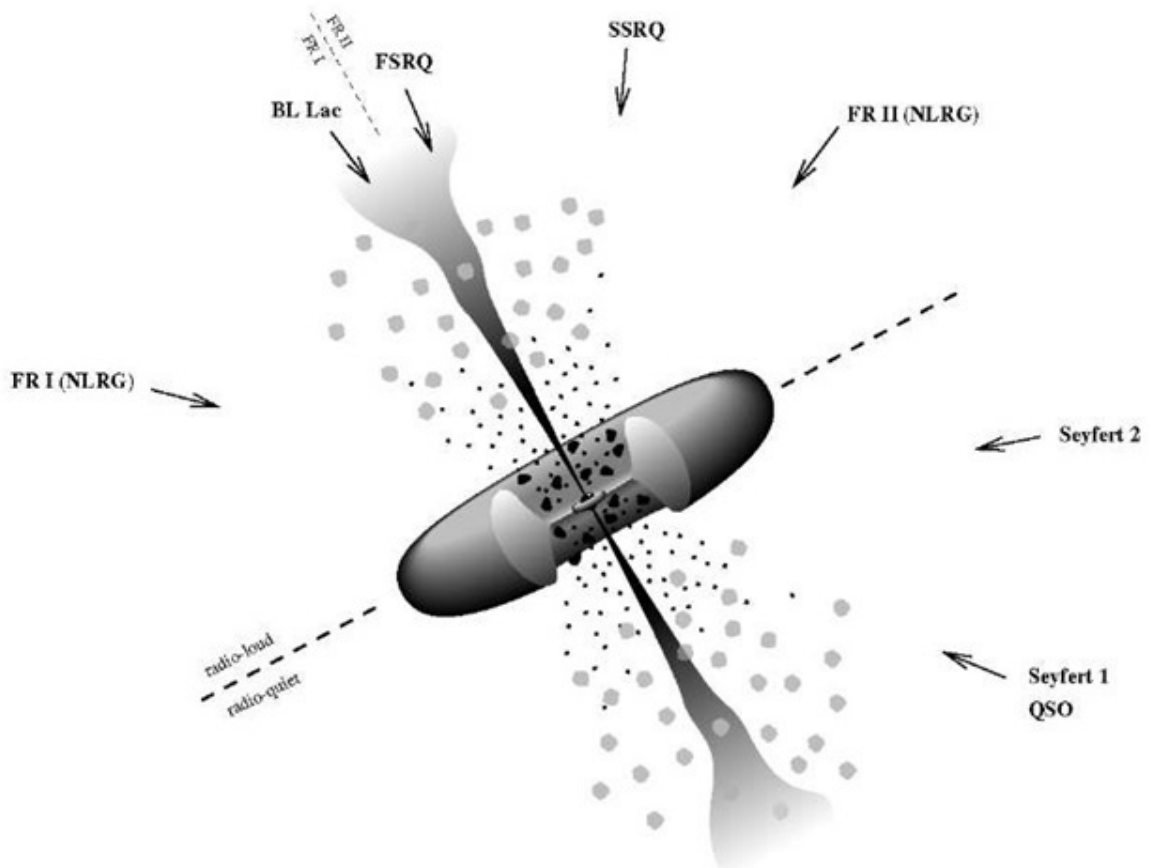

Fig. 2.- Classifications of AGN depending on their observational angle (Torres 2004). ${ }^{2}$

into two subclasses: Seyfert I and Seyfert II galaxies. Seyfert I galaxies have luminous nuclei and have strong emission in the blue optical continuum. They also have broad and narrow emission lines and are radio quiet. Furthermore, their narrow emission lines show high ionization. Seyfert II galaxies differ from Seyfert I galaxies in that they have weak emission lines and sometimes no blue optical continuum and only narrow emission lines are visible. Radio loud quasars make up another group of AGN and can be subdivided into broad line radio galaxies (BLRG) and narrow line radio galaxies (NLRG). Broad line radio galaxies are similar to Seyfert I galaxies and NLRG are similar to Seyfert II galaxies except that in both cases they are radio loud.

Another class of AGN is the QSO which combines objects such as BL Lac (and other similar objects) and optically violent variable (OVV) quasars. These objects are at an observational angle of nearly $0^{\circ}$ with respect to the jet, making the host galaxy difficult to

\footnotetext{
${ }^{2} \mathrm{http}: / /$ ned.ipac.caltech.edu/level5/March04/Torres/Torres2_4.html
} 
image. Steep spectrum QSOs show strong extended emission much more frequently than do flat spectrum QSOs. BL Lacertae objects show neither broad nor narrow emission lines. Their optical spectra show highly polarized continuum, are highly variable, exhibit strong radio emission and emit in the X-ray spectral region. OVV quasars show variability like BL Lac objects but otherwise have a normal QSO spectrum. Their continuum emission is highly variable and is thought to originate in a relativistic jet orientated close to the line of sight. BL Lac objects can be further classified as high-frequency BL Lac objects (HBL) and low-frequency BL Lac objects (LBL) on the basis of how much energy is contained in the peaks of their spectral energy distributions (SEDs). Blazars are the combined group of BL Lac objects and OVV quasars whose flux variability properties are described in detail in section 1.4.

\subsection{Blazar Variability}

Blazars include flat spectrum radio quasars (FSRQs) and BL Lac objects. Blazars have rapid high amplitude flux variability throughout the electromagnetic spectrum on all time scales, highly variable polarization, large beaming factors and high luminosities. The spectral energy distribution of blazars is usually a double peaked curve in which the low energy peak falls between the radio and x-ray region and the high energy peak falls in the $\gamma$-ray region. The low energy peak is attributed to the relativistic electrons in the jet producing synchrotron emission, whereas the high energy peak is attributed inverse-Compton emission due to the up-scatter of either the synchrotron photons or external photons by the relativistic electrons.

Initially the best models to explain relativistic jets were the synchrotron self-Compton (SSC) models (Marscher \& Gear 1985; Ghisellini \& Maraschi 1989). These models claim that the synchrotron mechanism produces the smooth, polarized and variable low energy continuum of blazar spectra whereas the Comptonization of synchrotron radiation 
is responsible for the high energy component. The Energetic Gamma Ray Experiment Telescope (EGRET) aboard the Compton Gamma Ray Observatory, discovered very high and rapid variable $\mathrm{MeV}-\mathrm{GeV}$ fluxes in many OVV quasars that exceeded synchrotron fluxes by factors of 10 or more (von Montigny et al. 1995). Thus an external photon field from either the accretion disk or broadline emission clouds might supply the scattered photons.

As previously stated, a characteristic of blazars is that their continuum emission is highly variable. This variability can be sub-categorized as long-term variability, short-term variability and microvariability. Long-term variability refers to variations that occur on timescales of months to years. Short-term variability refers to variations on timescales of weeks to months and microvariability refers to variations that occur on timescales of minutes to hours. Each type of variability gives insight into the specific physical processes in the jet and disk. For example, long-term variability is most likely the result of changing accretion rates onto the black hole, short-term variability to major shock wave events that occur in the jet, and microvariability to the inhomogeneity and turbulence within the jet (Marscher 2005). Since the jet of the blazar is closely aligned to the line of sight, the non-thermal radiation from the jet dominates the radiation from the accretion disk. Thus we assume that the variations are associated with physical processes in the jet, not in the disk. It is important to note that the variations seen in blazars are intrinsic and independent of location or atmospheric effects. It was shown that two telescopes at different locations, and with different CCD cameras and observing conditions, observe the same microvariations. (Pollock et al. 2007).

Short-term continuum variability could be due to particle acceleration in the blazar jet. Particle injection and particle acceleration at the base of the relativistic jet is often thought to be the cause of rapid variability at high energies $(\mathrm{GeV}-\mathrm{TeV})$. In this case, the radiation and kinetic energy of the particles are converted from the kinetic and internal energy of the jet itself. Another widely used model of particle acceleration in jets explains high 
amplitude short term flares by collisions of inhomogeneities ejected by the central engine with different bulk Lorentz factors and different velocities down the jet (Sikora, Begelman, \& Rees 1994). The collisions accelerate the particles and heat the electrons to medium energies which are then accelerated relativistically by shockwaves. "Microvariability" refers to low amplitude, extremely rapid continuum variations that must occur within the jet. The next section and the remainder of this thesis concentrates on models for these rapid microvariations. 


\section{Modeling Microvariability}

\subsection{AGN Jets}

As stated previously, the current model of most radio-loud AGNs consists of bipolar jets emanating from the central black hole. These jets can appear to be short or long, straight or curved, smooth or knotted and extend to several kilo-parsecs from the central core. These jets deposit power into the surrounding lobes (observed at radio wavelengths) (Begelman, Blanford, \& Rees 1984). Since the jets in blazars are viewed with nearly a $0^{\circ}$ viewing angle, beaming effects are strong and can therefore distort apparent geometry and show superluminal apparent speeds.

The jets are accelerated by differential rotation of the polodial component of the magnetic field in the ergosphere, or in the inner disk. The twisting magnetic fields emanating from the ergospehere most likely aid in collimating the jets (Meier, Koide, \& Uchida 2000). Active galactic nuclei variability is seen over the entire electromagnetic spectrum, and the intensities of the variations are amplified by relativistic beaming. To estimate the amount of energy in the jet, assumptions regarding the intensity of the magnetic field and amount of relativistic beaming in the jet must be made. The simplest assumption is that the energies are equally partitioned between the magnetic field and particle energy density.

Although the source is unresolved at high energies, we can resolve the compact radio knots that are synchrotron self-absorbed. This allows us to calculate the energy density in the magnetic field and the energy density in the radiating electrons separately. Perhaps the dominant process by which particles are injected into the base of the jet is through pair creation (Meier, Koide, \& Uchida 2000). Photons in the ergosphere of the central blackhole and accretion disk enter the jet and pair-create off the magnetic field. 


\subsection{Model}

To better understand the processes occurring in these jets we can try to model the continuum radiation and variations seen in blazars. In the present thesis we consider the shock-in-jet model to explain the optical variability properties seen in blazars. This model involves a shock passing though the jet and accelerating electrons to high energies. The shockwaves also compress the magnetic field, amplifying it, and the particles cool by interacting with the magnetic field and emitting synchrotron radiation. Marscher \& Gear (1985) tried to explain superluminal knots seen in radio wavelengths and X-ray variability observed in 3C 273 by claiming that they arise from shock waves passing through an adiabatic conical, relativistic jet. According to Sikora et al. (2001) these individual shock events are caused by velocity irregularities. Sokolov et al. (2004) claimed that the flares were a consequence of the collision of relativistic shocks with some stationary structures such as a Mach disk. A diagram of a turbulent jet from Marscher \& Gear (1985) is shown in Figure 3.

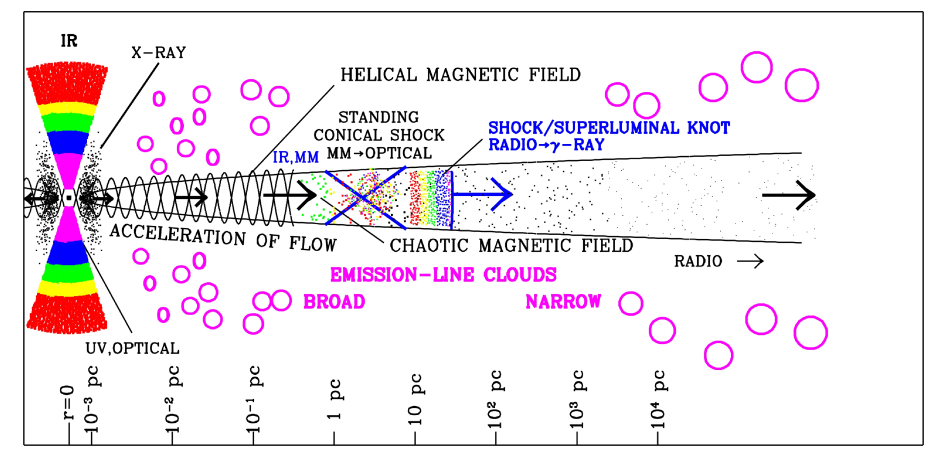

Fig. 3.- A model of a turbulent jet by Marscher \& Gear (1985).

As the shockwave travels through the turbulent plasma it excites individual cells, which then emit a pulse of synchrotron emission. The amplitude and shape of the pulse depends on the size of the cell, magnetic field and local density enhancement. However we note that if the flow of the plasma is laminar, there are no individual cells and we will see a flare but not microvariability (Bhatta et al. 2013). 


\subsection{Revised KRM Model}

To describe a short term flare seen in Mkn 501, Kirk, Rieger, \& Mastichiadis (1998) (hereafter KRM) modeled a shock wave passing through a cylindrical jet and interacting with a density-enhanced region of uniform magnetic field. The KRM model states that electrons are accelerated and emit synchrotron radiation as they cool. The duration of the pulse is therefore related to the size of the density-enhanced region. By revising this model slightly we can describe the microvariations seen in blazars. Instead of considering just one density-enhanced region as the KRM model did, we consider several density-enhanced regions modeled as cylindrical cells (see Figure 4).

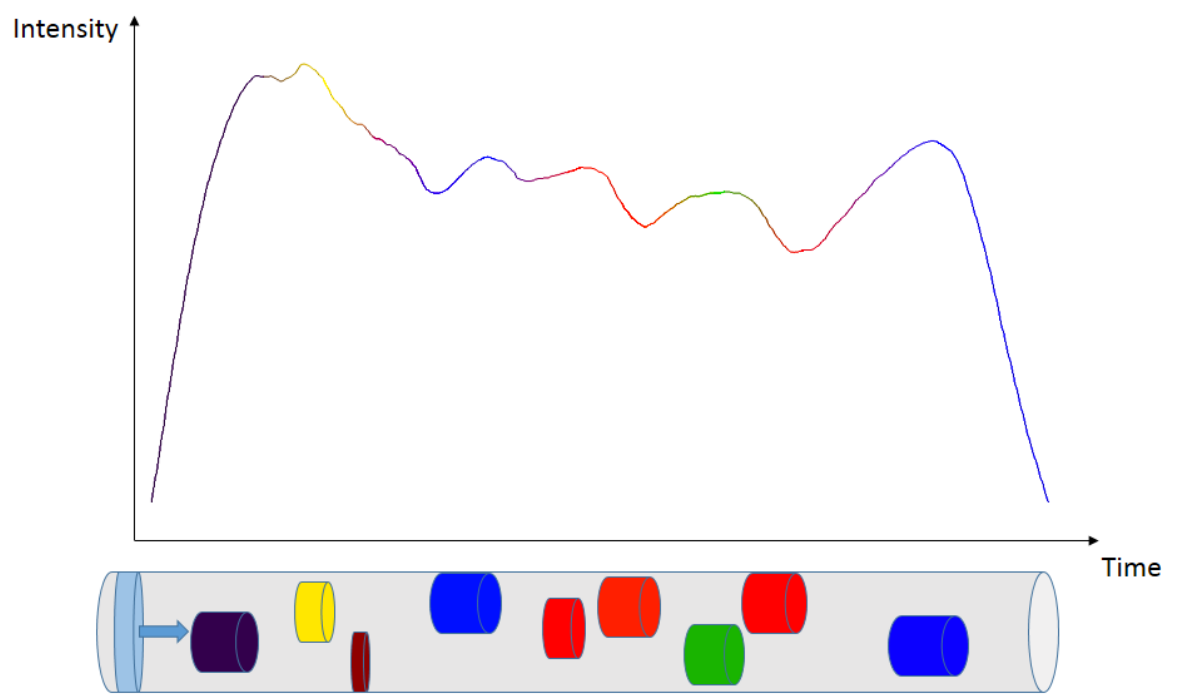

Fig. 4. - Revised KRM Model: Turbulent cells are modeled as cylinders filling the plasma jet. Cells of higher density are bluer and cells of lower density are redder. The shock front is shown as moving from left to right.

As the shock wave hits each individual cell, a pulse is generated whose duration is dependent on the size of the cell and whose amplitude depends on the density enhancement. To mathematically describe the KRM model, as done in Kirk, Rieger \& Mastichiadis (1998), we consider two regions of each cell: the acceleration zone and the emission zone. We consider the frame in which the plasma is at rest in the jet frame. 
The acceleration region is considered to be very small and occurs at the shock front which is modeled as a delta function. The diffusion equation in 2.1 describes the electron distribution when a plane shock wave hits electrons with energy $\gamma_{0}$ in the acceleration zone. The electrons are accelerated to a maximum energy $\gamma_{\max }$.

$$
\frac{\partial N}{\partial t}+\frac{\partial}{\partial \gamma}\left[\left(\frac{\gamma}{t_{a c c}}-\beta_{s} \gamma^{2}\right)\right] N+\frac{N}{t_{a c c}}=Q \delta\left(\gamma-\gamma_{0}\right)
$$

The first term on the left describes the change in number of particles $\mathrm{N}$, at a specific energy, over time and the third term is the escape term. The second term on the left (quantity in brackets) contains two terms: the acceleration term and the loss of energy due to synchrotron radiation, with $\beta$ given in equation 2.2 .

$$
\beta_{s}=\frac{4}{3} \frac{\sigma_{T}}{m_{e} c^{2}}\left(\frac{B^{2}}{2 \mu_{0}}\right)
$$

The term on the right side describes the particle injection rate. Kirk, Rieger \& Mastichiadis (1998) solved this differential equation using the Laplace transformation method to obtain the solution:

$$
N(\gamma, t)=a \frac{1}{\gamma^{2}}\left(\frac{1}{\gamma}-\frac{1}{\gamma_{\max }}\right)^{\left(t_{a c c}-t_{e s c}\right) / t_{e s c}} \times \Theta\left(\gamma-\gamma_{0}\right) \Theta(\gamma 1(t)-\gamma)
$$

This is the solution given certain boundary conditions $\left(\gamma_{0}<\gamma<\gamma_{1}(\mathrm{t})\right.$ and $\left.\mathrm{N}(\gamma, \mathrm{t})=0\right)$, where $\Theta$ is the Heaviside step functions and $a$ is a constant:

$$
a=Q_{0} t_{a c c} \gamma_{0}^{t_{a c c} / t_{e s c}}\left(1-\frac{\gamma_{0}}{\gamma_{\max }}\right)^{-t_{a c c} / t_{e s c}}
$$

If one considers that the electron begins at some energy $\gamma_{0}$, then after a certain time $t$, the electron may not have reached $\gamma_{\max }$, but instead reaches $\gamma_{1}(\mathrm{t})$ :

$$
\gamma_{1}(t)=\left(\frac{1}{\gamma_{\max }}+\left[\frac{1}{\gamma_{0}}-\frac{1}{\gamma_{\max }}\right] e^{-t / t_{a c c}}\right)^{-1}
$$


As particles are accelerated, they travel downstream in the plasma where they cool by emitting synchrotron radiation. This therefore is considered to be the emission zone and is restricted to length $L$, the maximum extent of the emission region,

$$
L=\frac{t_{b} u_{s}}{1-u_{s} / c}
$$

Just as there was a diffusion equation to describe the particle density in the acceleration zone, there is a similar equation to describe the particle density in the emission zone, however this time there is no acceleration term.

$$
\frac{\partial n}{\partial t}-\frac{\partial}{\partial \gamma}\left(\beta_{s} \gamma^{2} n\right)=\frac{N(\gamma, t)}{t_{e s c}} \delta\left(x-x_{s}(t)\right)
$$

Here $n$ is the number density per unit length for a given energy and $x_{s}(\mathrm{t})$ is the position of the shock front. Following a similar procedure as done in the acceleration zone, if we solve the differential equation for the emission zone using the Greens synchrotron function,

$$
P(\nu, \gamma)=a_{s} z^{0.3} \exp (-z)
$$

where $a_{s}=\frac{\sqrt{3} e^{2} \Omega \sin \theta}{2 \pi c}$ is a constant, and $z \equiv 4 \pi \nu /\left(3 \Omega \sin \theta \gamma^{2}\right)$, with $\Omega=\frac{e B}{m}$ the electron gyro frequency, and $\theta$ the angle between the magnetic field direction and the line of sight, we get the solution

$$
\begin{aligned}
n(x, \gamma, t)=\frac{a}{u_{s} t_{e s c} \gamma^{2}} & {\left[\frac{1}{\gamma}-\beta_{s}\left(t-\frac{x}{u_{s}}\right)-\frac{1}{\gamma_{\max }}\right]^{\left(t_{a c c}-t_{e s c}\right) / t_{e s c}} } \\
\times & \Theta\left[\gamma 1\left(x / u_{s}\right)-\left(1 / \gamma-\beta_{s} t+\beta_{s} x / u_{s}\right)^{-1}\right] .
\end{aligned}
$$

Here we have used boundary conditions such that acceleration begins at $\mathrm{x}=0$ and $\mathrm{t}=0$, and that the shock moves with constant speed $u_{s}$. This describes the emission at a particular energy. In order to determine the total intensity of the emission we must first integrate the 
intensity for a single energy and single time, over the spatial volume.

$$
\int n(x, \gamma, t) d x=\frac{a}{\left(1-\frac{u_{s}}{c}\right) \gamma_{(\max )}^{\left(t_{a c c}+t_{e s c}\right) / t_{e s c}}}\left(\frac{\gamma_{\max }}{\gamma}\right)^{2}\left\{\left[\frac{\gamma_{\max }}{\gamma}-\frac{t}{t_{a c c}}+\frac{x\left(1-u_{s} / c\right)}{u_{s} t_{a c c}}\right]^{t_{a c c} / t_{e s c}}\right\}_{x_{0}(\gamma, t)}^{x_{1}(t)}
$$

Equation 2.11 is an expression for the synchrotron radiation emitted while the particle is in the emission zone. However we must also consider the contribution to the intensity given by the particle while it is in the acceleration zone.

$$
I_{0}(\nu, t)=\iint P(\nu, \gamma) n(x, \gamma, t+x / c) d x d \gamma
$$

If the magnetic field in front of the shock front was the same as the magnetic field after the shock front then we can just simply use 2.11. However for the $t>t_{\text {on }}$ (where $t_{\text {on }}$ is the time the shock begins), we have an extra term

$$
\begin{array}{r}
I_{s}(\nu, t)=\int d \gamma P(\nu, \gamma) \int d x N(\gamma, t) \delta\left[x-u_{s}(t+x / c)\right]= \\
\frac{a}{\left(1-\frac{u_{s}}{c}\right) \gamma_{(\max )}^{\left(t_{a c c}+t_{e s c}\right) / t_{e s c}}} \int d \gamma P(\nu, \gamma)\left(\frac{\gamma_{\max }}{\gamma}\right)^{2}\left(\frac{\gamma_{\max }}{\gamma}-1\right)^{\left(t_{a c c}+t_{e s c}\right) / t_{e s c}} .
\end{array}
$$

Therefore the total emission would be given by

$$
I(\nu, t)=I_{0}(\nu, t)+I_{s}(\nu, t) .
$$

If we now would like to model variability we can increase the particle injection rate by a factor of $1+\eta_{f}$ for a time $t_{f}$. We then have that

$$
\begin{gathered}
Q(t)=Q_{0} \text { for } t<0 \text { and } t>t_{f} \text { and } \\
Q(t)=\left(1+\eta_{f}\right) Q_{0} \text { for } 0<t<t_{f}
\end{gathered}
$$


Our total intensity now becomes

$$
I(\nu, t)=I_{1}(\nu, t)+\eta_{f}\left[I_{1}(\nu, t)-I_{1}\left(\nu,\left(1-u_{s} / c\right) t_{f}\right)\right]
$$

We used the computer language IDL by Exelis to write a program to solve the above equation. The pulse program calculates and plots the shape of the curve (Figure 5) resulting from the equations above (Bhatta et al. 2013). The pulse program in IDL is included as Appendix I. As seen in Figure 5, the shape of the pulse is dependent on the frequency of the emission.

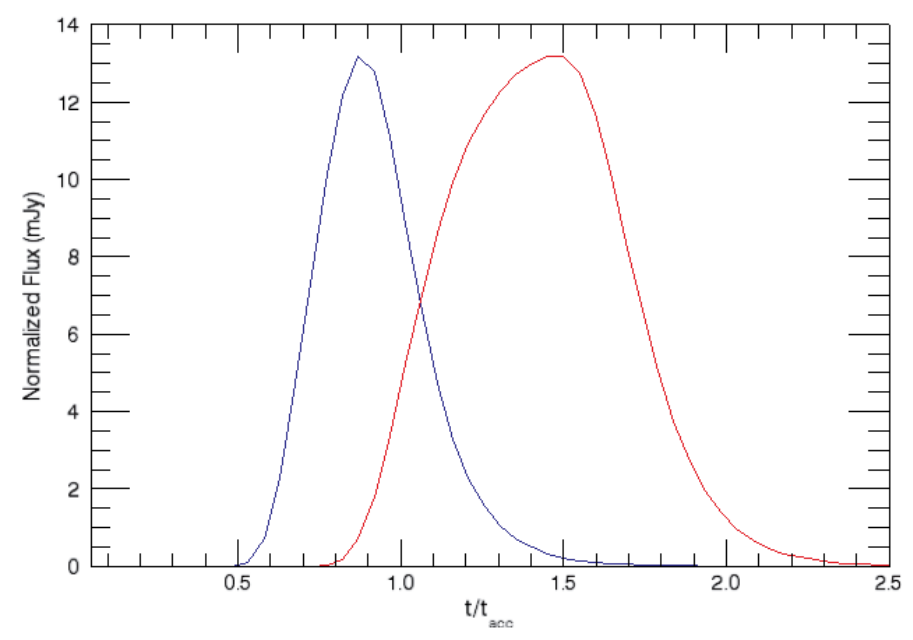

Fig. 5.- Pulse shapes for two different frequencies: $\nu=4.3 \times 10^{16} \mathrm{~Hz}$ (blue) and $\nu=$ $4.3 \times 10^{17} \mathrm{~Hz}$ (red). Input parameters : $B=2$ Gauss, $Q=609.3 \mathrm{~m}^{-3} \mathrm{~s}^{-1}$ and time of flare $t_{f}=1.25 / t_{a c c}$.

The size of the cell can then be computed by using the width of the pulse:

$$
\begin{array}{r}
\text { cellsize }=t_{\text {flare }} \times \beta c \\
t_{\text {flare }}=\frac{1}{2} \times \text { pulsewidth } .
\end{array}
$$

The model also predicts the behavior of the spectral index as the flare evolves. The spectral index is a measure of the dependence of flux on frequency. If the information 
about changes in injection propagates from high to low energies by synchrotron cooling (or any other cooling method), the spectral index exhibits a clock-wise loop-like pattern when plotted against the flux at the lower frequency (Figure 6). This pattern has been observed by several sources in different wavelengths e.g. OJ 287 (Gear et al. 1986), Mkn 421 (Takahashi et al. 1996; Kirk, Rieger \& Mastichiadis 1998). If the spectral index is plotted against the higher frequency observed where cooling and acceleration times are equal, the loop-like behavior is still present except that the direction is now counter clock-wise (Figure 7).

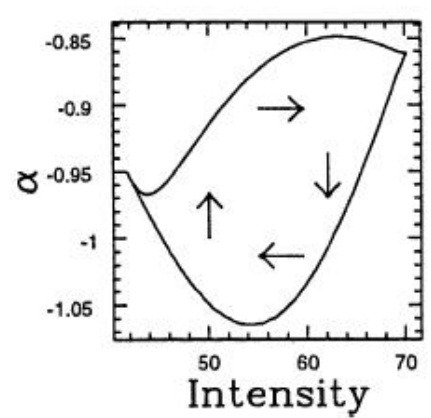

Fig. 6.- Clockwise hysteresis loop produced when spectral index is plotted against flux of lower frequency (Kirk, Rieger \& Mastichiadis 1998).

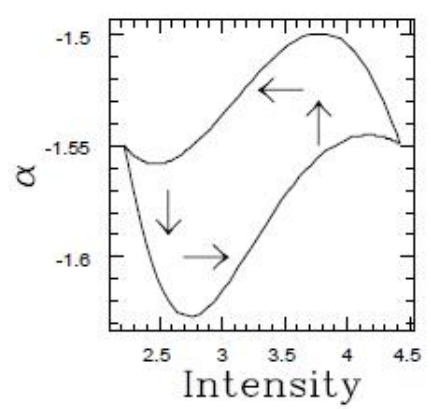

Fig. $\quad$ 7.- CounterClockwise hysteresis loop produced when spectral index is plotted against flux of higher frequency (Kirk, Rieger \& Mastichiadis 1998).

Before we can quantify luminosities or sizes in the source rest frame, we must consider that these objects are at a very high red shift and the jet speeds are relativistic, so relativistic corrections need to be made using a revised Doppler Factor

$$
\text { Correction Factor }=\frac{z}{\Gamma(1-\beta \cos \varphi)}
$$

(Kembhavi \& Narlikar 1999) where $\Gamma$ is the Lorentz bulk factor, $\beta$ is the velocity, $\varphi$ is the angle between the jet and the line of sight and $z$ is the redshift. 


\subsection{Previous Data}

The first test to the revised version of the KRM model was done on the 2009 Whole Earth Blazar Telescope (WEBT) campaign. For this campaign, Bhatta et al. (2013) collaborated with 36 observatories in 16 countries to create a 78.88 hour light curve of $0716+71$. The details of the campaign are listed in Table 1 .

The short term and long term trend in the light curves were subtracted so that only microvariations remained. The location of each pulse was determined to be the center of each local peak in the data and its amplitude and size were determined by a best fit to the data. Bhatta et al. (2013) fit 35 pulses to the light curve with a correlation coefficient of 0.98 , showing that the model correctly described the light curve behavior. However because the WEBT data were only taken in a single filter, the R filter, they were unable to test the hysteresis loop change in the spectral index.

Rafle et al. (2012) simultaneously conducted a microvariability study to test the KRM model using data from the long term monitoring campaign of $0716+71$ by Montagni et al. (2006) from 1996-2003. Rafle et al. (2012) fit pulses to 37 individual light curves, each with an average duration of nine hours. Each light curve had an average of 6.43 pulses and a maximum and minimum correlation coefficient of 0.997 and 0.85 , respectively. Similarly, the spectral index test was not able to be completed since there were no simultaneous multi-frequency observations available.

Table 1 WEBT Campaign Data

\begin{tabular}{lc}
\hline \hline Time Length of Campaign & $78.88 \mathrm{hrs}$ \\
Number of Data Points & 2613 \\
Mean Magnitude (R) & 13.75 \\
Amplitude & 0.31 \\
Standard Deviation & $9.8 \%$ \\
\hline
\end{tabular}




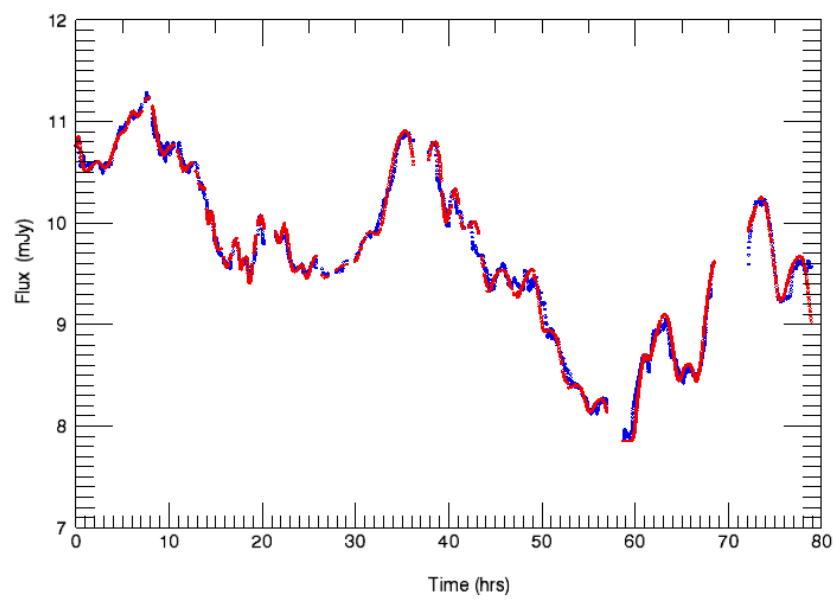

Fig. 8.- Results of the 2009 WEBT Campaign (Bhatta et al. 2013).

In order to verify that the KRM correctly describes the microvariations seen in blazars, simultaneous multi-frequency observations need to be made in order to determine if the spectral index changes with time in a hysteresis loop manner predicted by the model. For this we require simultaneous observations of the same object with two different filters. The spectral variability analysis is detailed further in chapter 3 .

In addition to testing spectral variability, the pulse model needs to be tested on more than just one object. We have data on several objects taken with the Southeastern Association for Research in Astronomy ${ }^{3}$ (SARA) 0.9-m telescope, however these data posed a few problems. Because the duration of a night is a maximum of about nine hours and because the uncertainty in the flux was at times large, we were unable to determine whether the pulses from SARA data were actually one pulse or two convolved pulses. Another problem is the relatively large error in the SARA data compared to the expected effect.

\footnotetext{
${ }^{3}$ Southeastern Association for Research in Astronomy (http://saraobservatory.org/).
} 


\section{Multi-color Study of $0716+71$}

In order to further test the KRM model, we carried out an attempt to test for the frequency hysteresis predicted by the KRM model. This part of our study focused on the spectral index analysis of $0716+71$. Blazar $0716+71$ is a bright BL Lac object with a redshift of $\mathrm{z}=0.31 \pm 0.08$. This object was chosen since it has a duty cycle of about $95.3 \%$, meaning it is nearly always undergoing microvariability (Webb et al. 2010). Since we only needed one clearly isolated pulse to produce the expected spectral hysteresis, we were able to use the SARA $0.9 \mathrm{~m}$ telescope located in Kitt Peak, Arizona. The observations were taken in Johnson \& Cousins V and I bands simultaneously. The bands were chosen since they are the most widely separated of the optical frequency bands to which our cameras are sensitive. The simultaneous light curves are shown in Figure 9 and 10.

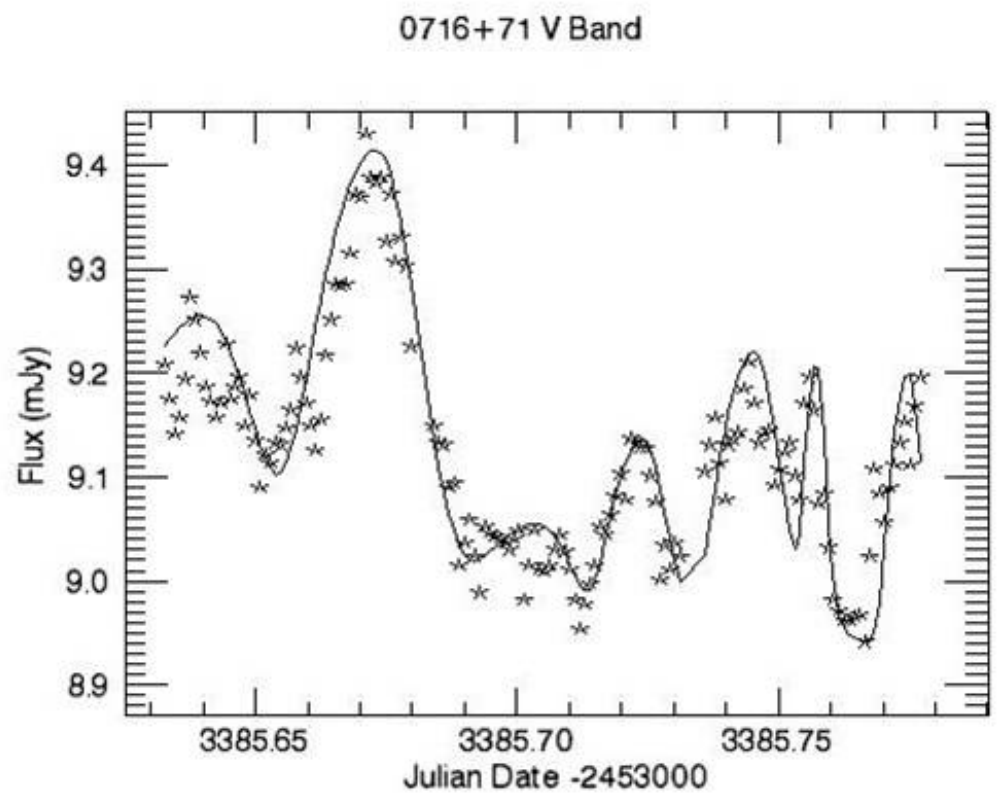

Fig. 9. $-\mathrm{V}$ band light curve of $0716+71$ fitted with 7 pulses. Data taken on 4/03/13 with SARA 0.9-m telescope.

Finally, spectral variability was plotted against $\mathrm{V}$ band flux for one isolated pulse (Figure 11). Analysis showed there was no smooth hysteresis loop as predicted by the 


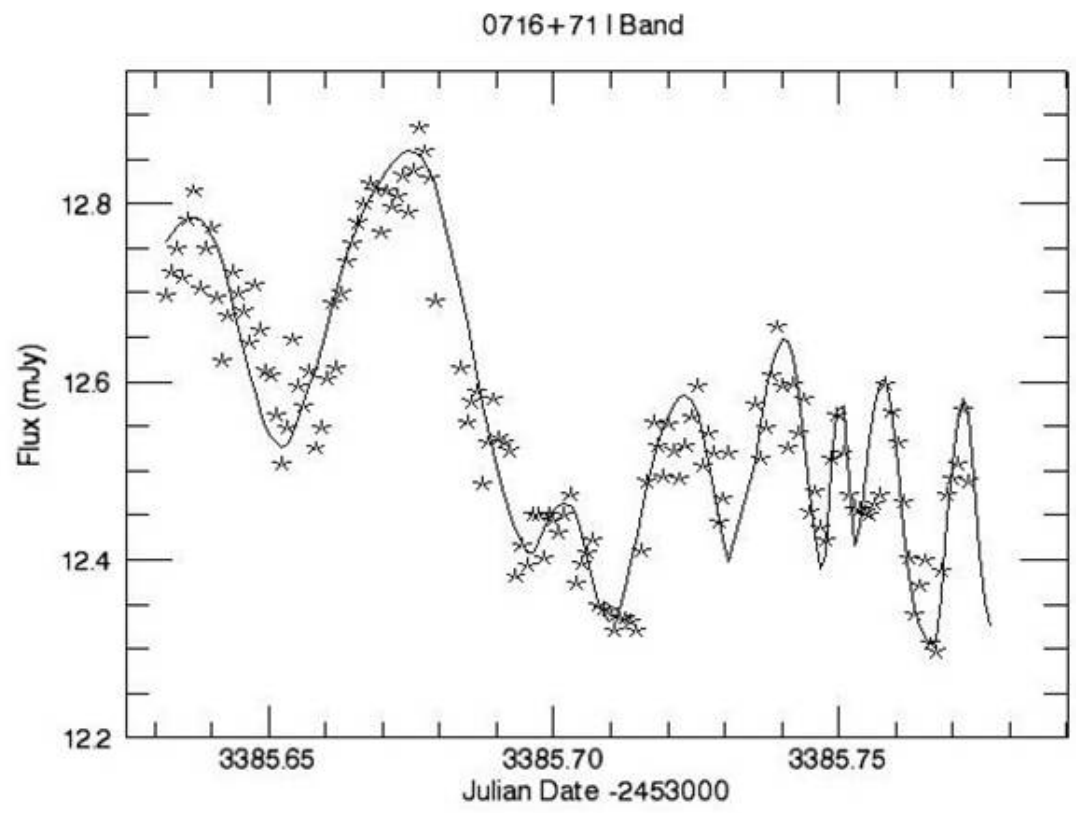

Fig. 10. - I band light curve of $0716+71$, fitted with 8 pulses. Data taken on 4/03/13 with SARA 0.9-m telescope.

KRM model, although there was a loop structure (beginning at $9.0 \mathrm{mJy}$ and looping counter clockwise to $9.12 \mathrm{mJy}$ ). Not much confidence can be placed in the loop structure seen in the data since the error in flux was quite large. Perhaps the bands are not as separated enough in frequency to produce the spectral variability within error. As seen in Figure 12, the pulse shape for the V and I bands are not dramatically different. In the timescale of this thesis, we were unable to gather simultaneous data from two widely separated frequency bands. 


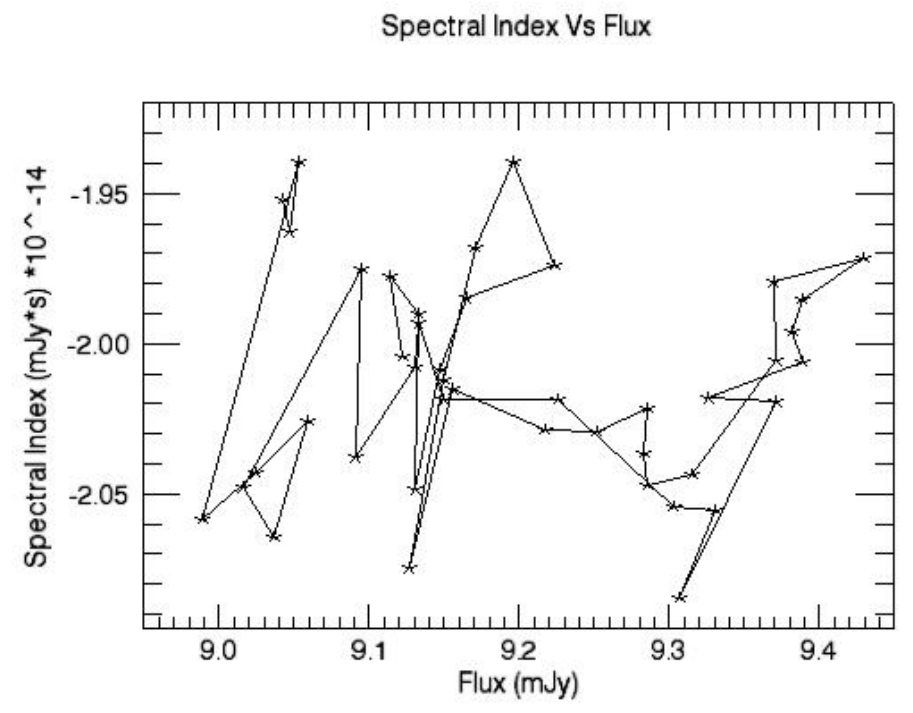

Fig. 11.- Spectral index vs. V band flux.

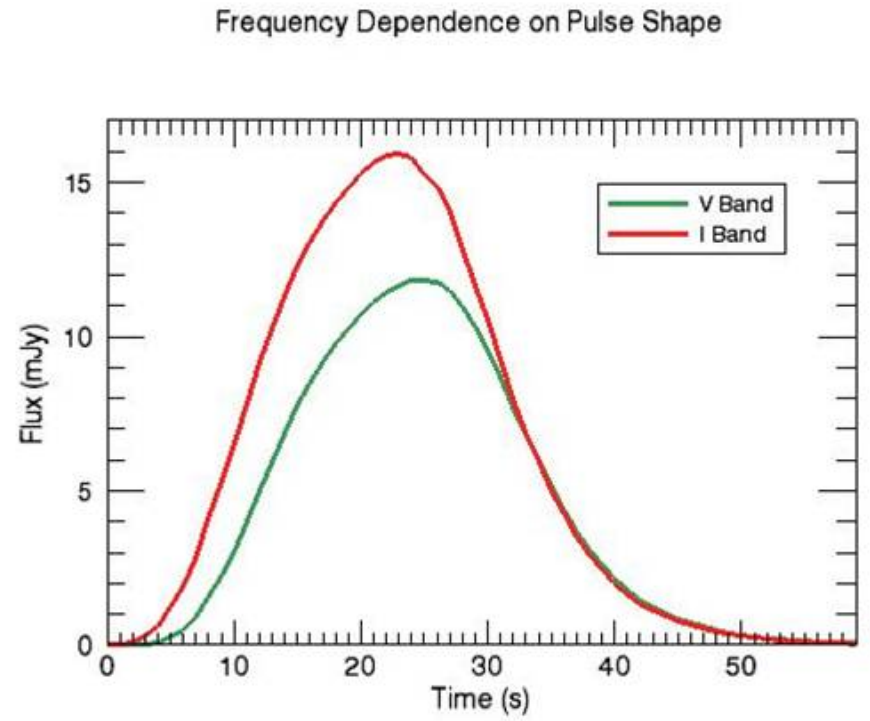

Fig. 12.- V (green) and I (red) band pulse shape. 


\section{The Kepler Mission}

\subsection{Kepler Space Telescope}

An additional test of the KRM model is fitting long, continuous and high quality data sets. In 2010, the Kepler satellite telescope observed four AGN intensively and with high precision. On March $7^{\text {th }}, 2009$, NASA launched Kepler into an Earth-trailing heliocentric orbit. Kepler's primary mission was to detect transiting Earth-size planets in the habitable zone of $\mathrm{F}$ through $\mathrm{M}$ type stars in the constellations Lyra and Cygnus. These data sets spanned three months each and are excellent candidates for our study. Since Kepler was trying to detect planetary transits, which only last a fraction of a day, it was important that the field of view was not blocked during any time of the year. Furthermore, it was important to have a large number of stars in the field of view (FOV), while at the same time not be on the galactic plane in order to reduce field confusion. This led to the field of view being large enough to monitor $\sim 100,000$ stars in the constellations Cygnus and Lyra (Figure 13). Every quarter, Kepler rolled 90 degrees about its optical axis in order to keep the sun on the solar panels and keep the radiators pointed to deep space to cool the focal plane. This meant that although the same area of the sky was observed, a particular star could be in one of four different parts of the focal plane depending on the season (Van Cleve et al. 2009).

Kepler has a $0.95-\mathrm{m}$ aperture Schmidt telescope with a 94.6 million pixel CCD detector array, which contains both the Science and Fine Guidance Sensor (FGS) pixels. The diameter of the FOV is $115.6 \mathrm{FOV}$ is $115.6 \mathrm{deg}^{2}$ and is covered with active pixels. The "frame" is considered the set of pixels collected by one repetition of the CCD clocking cycle and the integration time is the interval between reads of a given pixel. Observing periods are defined as "cadences" and are the set of co-added and stored pixels over a certain amount of time. Kepler collects two main types of cadences: short cadence and long cadence. The default exposure time for a short cadence is 


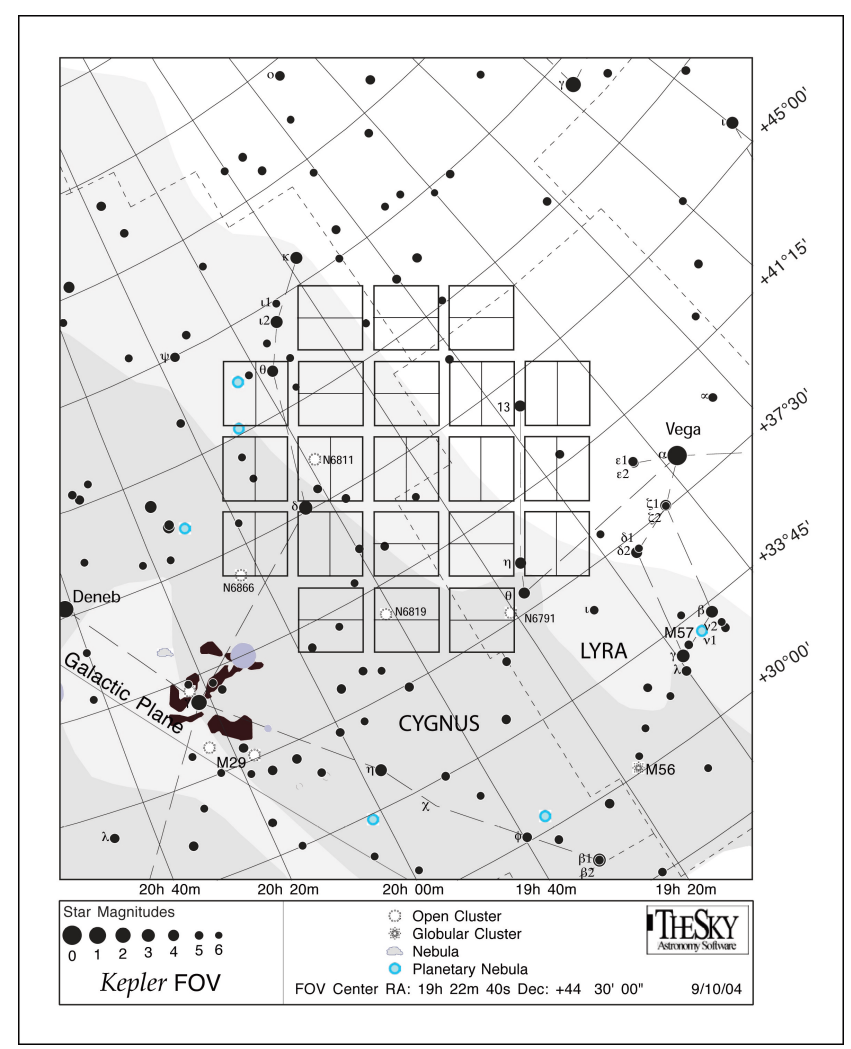

Fig. 13. - Kepler's field of view, centered in the constellations Lyra and Cygnus (Van Cleve et al. 2009).

54.2 seconds whereas the default exposure time for a long cadence is 1626 seconds (https://archive.stsci.edu/mast_faq.php?mission=KEPLER).

Every month, data are downlinked through the High Gain Antenna (HGA) and processed by the Data Management Center (DMC) and then archived at the Space Telescope Science Institute (STScI). Data calibration and download is discussed in more detail in the next section, however it is important to note that Kepler does not download full images. Furthermore, since Kepler is designed to repeat observations precisely it does not have many user options. There are no filters or integration parameters that a user can select and only Kepler magnitudes and counts (e/second) are recorded. Instrument calibration and long-term operating parameters were fixed during the Commissioning Phase which was concluded on May $12^{\text {th }}, 2009$ (Van Cleve et al. 2009). 


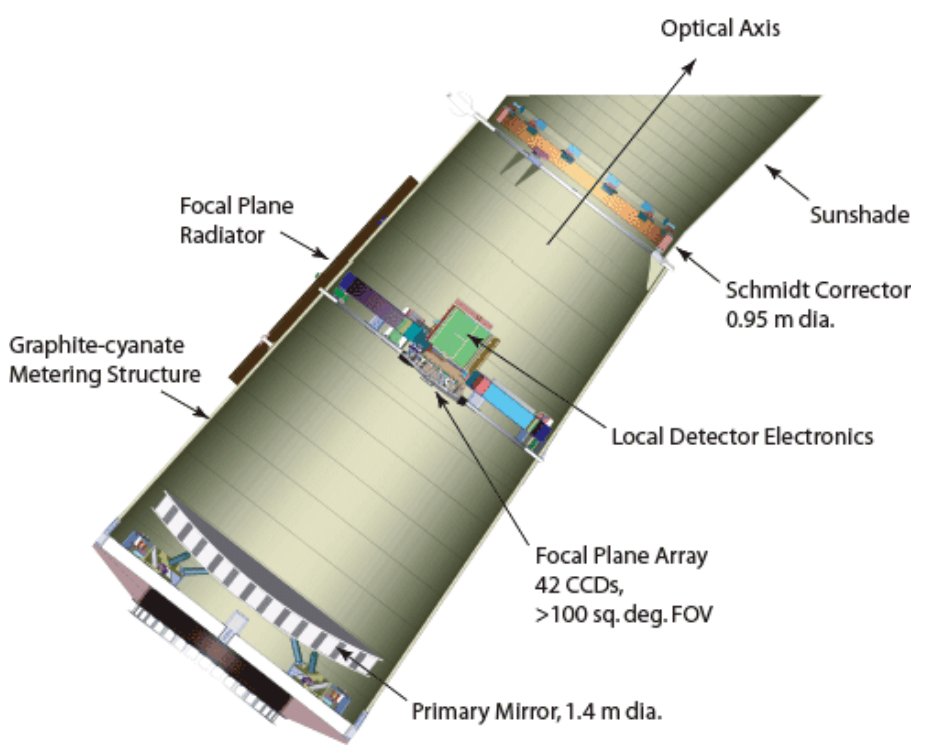

Fig. 14. - Kepler spacecraft (Van Cleve et al. 2009).

Initially Kepler was supposed to last 3.5 years, but as a result of interference from the spacecraft, additional time was needed to complete the mission. The extended mission was supposed to last until 2016, however on May $11^{\text {th }}, 2013$ a second of four gyroscope-like reaction wheels failed, meaning the current mission needed to be modified. "K2," also known as "Second Light", is a newly proposed plan presented in November 2013. K2 would use Kepler's remaining capability to collect data on supernovae, young and old stars, and AGN. The orientation of the spacecraft must be nearly parallel to its orbital path around the sun to achieve necessary stability. Instead of the four rolls per year as before, K2 would study a specific portion of the sky for 83 days and turning 4.5 times per year (http://www.nasa.gov/kepler/keplers-second-light-how-k2-will-work/\#.Uz2Z9vldV0Y).

In addition to the 2,740 planetary candidates, Kepler also found 3 new AGN (KA 1904+37, KA 1925+50, KA 1858+48) in its initial phase. These new, serendipitously discovered AGN happened to lie in the observed field, along with AGN Zw 229-15, and had well-observed light curves. After it was realized the Kepler had observations of these four AGN, several analyses of their light curves were conducted which are further described in section 5.1. 


\subsection{Kepler Data Pipeline}

The science pipeline consists of several steps. Once every month, data are transmitted from the spacecraft to the Mission Operations Center, located at the Laboratory for Atmospheric and Space Physics (LASP) in Boulder, Colorado and then to the DMC. The data are then sent to the Kepler Science Operations Center (SOC). The pipeline consists of several steps and allows for parallel processing of the data. First, raw pixel data are downlinked to the Calibration module (CAL). This produces calibrated pixels of the target object and the background. The data are then processed by Photometric Analysis (PA), which removes sky background and extracts simple aperture photometry. Before a light curve is created, the data go through Pre-search Data Conditioning (PDC). The pre-search data conditioning step removes systematic errors such as pointing drift, focus changes, and thermal transients and is specific to the detection of extra-solar planets (Jenkins 2010).

Kepler has unprecedented data quality with its $\lesssim 0.1 \%$ repeatability. A sample light curve of Zw 229-15 is shown with error bars in Figure 15. An independent check of Kepler data was done by the ground-based Lick AGN Monitoring Campaign (LAMP) for $\mathrm{Zw}-229-15$. The light curves show very good agreement between Kepler and LAMP within the $1 \%$ errors of LAMP. Kepler errors are however much smaller than LAMP errors and currently there is no way to be sure that systematic errors are not affecting the data. There are small short-term discontinuities following monthly downloads that are believed to be due thermally induced focus changes (Mushotzky et al. 2011).

Two types of flux measurements are recorded, PDC SAP FLUX and SAP FLUX. The differences between these two measurements lie in the correction factor for some of the short-term gain effects. Since PDC SAP FLUX count rates are identical to the SAP FLUX count rates only offset by a constant, the variations are identical in each set (Figure 16). We use the SAP FLUX rates in our analysis so as not to remove any small variations that may have been removed by the PDC processing, but are intrinsic to the quasar. 

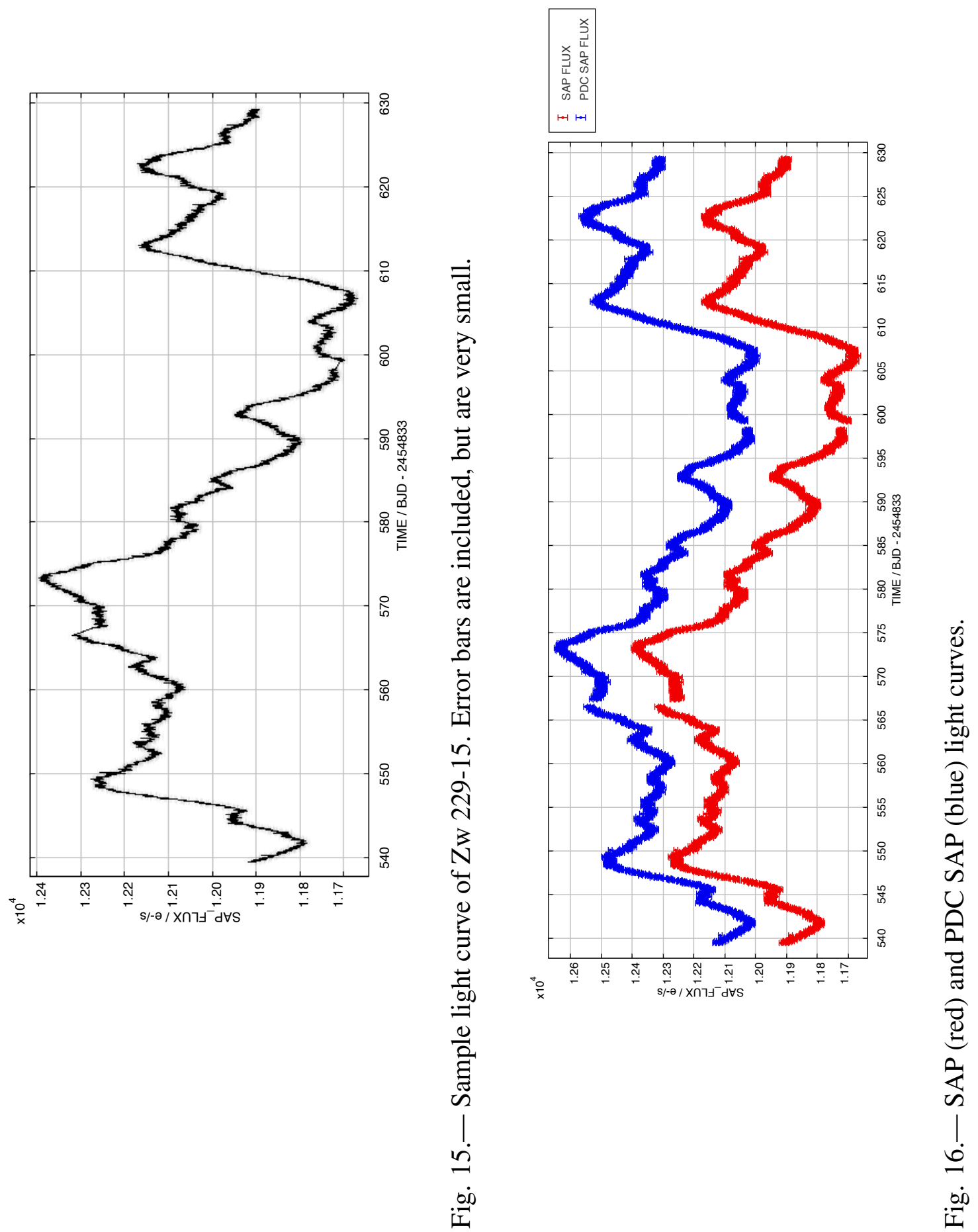


\section{Data Analysis}

\subsection{Pulse Fitting}

Because Kepler has such large temporal coverage, sampling one point every 30 minutes, and excellent data quality, we are able to use the Kepler data sets to study the microvariability in the four Kepler AGN (Table 2) in our microvariability study. Because of the flux discontinuity, these data sets cannot be combined into one large data set, however each quarter contains 3 months of continuous data which is a factor of 10 longer than even the WEBT $0716+71$ observation.

In this thesis, we have applied the modified KRM jet model to the variations seen in the Kepler AGN data. Because the Kepler data sets are of such high quality, we can use the KRM model to analyze the properties of the Kepler AGN jets through the microvariability pulse analysis. Recall that Kepler has no filters, therefore the "Kepler magnitude" is defined as the spectral response of the CCD convolved with the color of the target (Van Cleve et al. 2009). At present, there is no reliable conversion from Kepler magnitudes to the standard Johnson \& Cousins magnitude system used in other light curves. We modeled the pulse shape based on the peak spectral response of Kepler which is centered on 575 nm. The resulting pulse shape is shown in Figure 17.

In order to fit the pulses to the data, we used the IDL pulse fitting program, documented in Appendix II. In our model, each pulse corresponds to the shockwave hitting a density-enhanced region within the jet. By convolving the pulses, we treat the light curve as a convolution of pulses from the turbulent cells that vary in size, density, and location within the jet. We take the center of the pulse to be the center of each individual feature seen in the light curve and then adjust the Full Width Half Max (FWHM) of the pulse, retaining the shape, to fit the feature. 
Table 2 Kepler AGN Source Information

\begin{tabular}{lccc}
\hline \hline Source Name & RA (J2000) & $\operatorname{Dec}(\mathrm{J} 2000)$ & $\mathrm{Z}$ \\
\hline Zw 229-15 & 190526.0 & +422740 & 0.028 \\
KA 1925+50 & 192502.2 & +504314 & 0.067 \\
KA 1858+48 & 185801.1 & +485023 & 0.079 \\
KA 1904+37 & 190458.7 & +375541 & 0.089 \\
\hline
\end{tabular}

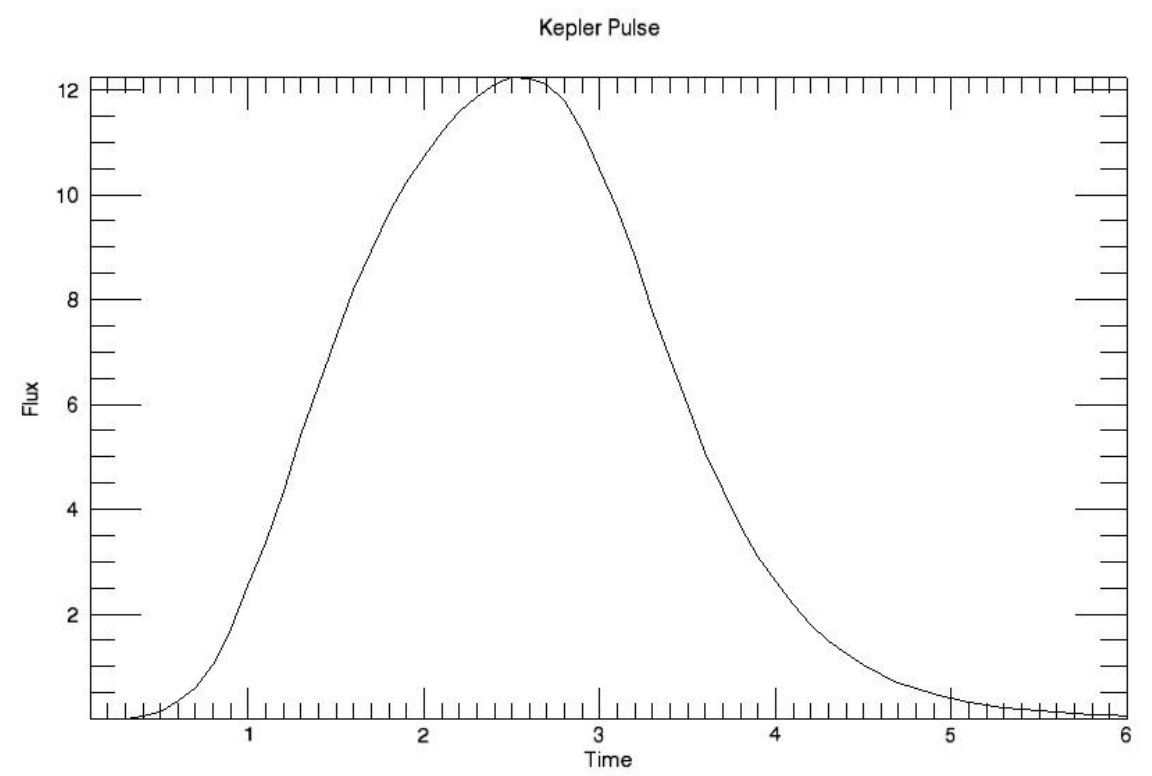

Fig. 17. - Shape of the Kepler pulse (frequency=575 nm). Time and Flux units are arbitrary in this figure. 
The center of the cell therefore gives us the location of the cell, the amplitude corresponds to the density enhancement within the cell, and the pulse width to the size of the cell. The best fit to the data was determined by using a combination of the FWHM and amplitude that produced the highest correlation coefficient to the data. Cadences 6 and 7 were chosen for each object (except for KA 1904+37 because no cadence 6 information was available) for the analysis. Details of the pulses fitted for each object are shown in Appendix III. Table 3 summarizes the analysis giving the cadence number, number of points in each data set, number of pulses fit, average cell size (AU), correlation coefficient and degrees of freedom ${ }^{4}$. Errors are not noted, however fits were performed until the correlation coefficient was highest.

\footnotetext{
${ }^{4}$ The degrees of freedom are defined as $(\#$ points $-(\#$ pulses $\times 3)-1)$.
} 
Table 3 Pulse Fitting Data

\begin{tabular}{ccccccc}
\hline \hline $\begin{array}{c}\text { Galaxy Name } \\
\text { Name }\end{array}$ & $\begin{array}{c}\text { Cadence } \\
\text { Number }\end{array}$ & $\begin{array}{c}\text { Number } \\
\text { of Points }\end{array}$ & $\begin{array}{c}\text { Number of } \\
\text { Pulses/Cells }\end{array}$ & $\begin{array}{c}\text { Average Cell } \\
\text { Size (AU) }\end{array}$ & $\begin{array}{c}\text { Correlation } \\
\text { Coefficient }\end{array}$ & $\begin{array}{c}\text { Degrees } \\
\text { of Freedom }\end{array}$ \\
\hline \multirow{2}{*}{ Zw 229-15 } & 6 & 4276 & 38 & 35.83 & 0.98 & 4161 \\
\hline \multirow{2}{*}{$1925+50$} & 7 & 4227 & 42 & 34.65 & 0.99 & 4100 \\
\hline \multirow{2}{*}{$1858+48$} & 6 & 4277 & 55 & 36.70 & 0.99 & 4111 \\
& 7 & 4226 & 52 & 40.34 & 0.99 & 4069 \\
\hline $1904+37$ & 6 & 4277 & 46 & 31.34 & 0.99 & 4138 \\
\hline \hline
\end{tabular}




\subsubsection{Zw 229-15}

The cadence 6 and 7 light curves for $Z w 229-15$ are shown in figures 18 and 19. The cadence 6 light curve had a total of 4,276 points and we fit 38 pulses with a correlation coefficient of 0.985 and 4161 degrees of freedom. Cadence 7 had a total of 4,227 points with a total of 42 pulses and a correlation coefficient of 0.99 and 4100 degrees of freedom. The average cell size for cadence 6 was $35.82 \mathrm{AU}$ and for cadence 7 was $34.65 \mathrm{AU}$. The frequency distribution of cell sizes is shown in Figure 20. The figure clearly shows that most cells are of a smaller size consistent with the distribution of cell sizes expected in a turbulent plasma.

\subsubsection{KA $1925+50$}

For object KA $1925+50$, cadence 6 had a total of 4,277 points and 55 pulses and a correlation coefficient of 0.99 . Similarly, cadence 7 had a total of 4,226 points and 52 pulses total. The average cell size for KA $1925+50$ is similar to that of $Z w$ 229-25. Again, the cell size frequency distribution is consistent with the turbulent plasma assumption (Figure 23).

\subsubsection{KA $1858+48$}

Fitted light curves for KA 1858+48 cadence 6 and 7 are shown in Figure 24 and 25, respectively. Again cell sizes seem to be consistent with the first three objects in our study and the cell size frequency distribution is therefore consistent with the cell size distribution for a turbulent plasma. 


\subsubsection{KA $1904+37$}

The last object in our study, KA 1904+37 did not have data during cadence 6 so only cadence 7 was analyzed. KA $1904+37$ had a total of 27 pulses in 4,227 points. The cell size frequency distribution is similar to the other objects, with most cells ranging 40-60 AU in size. 


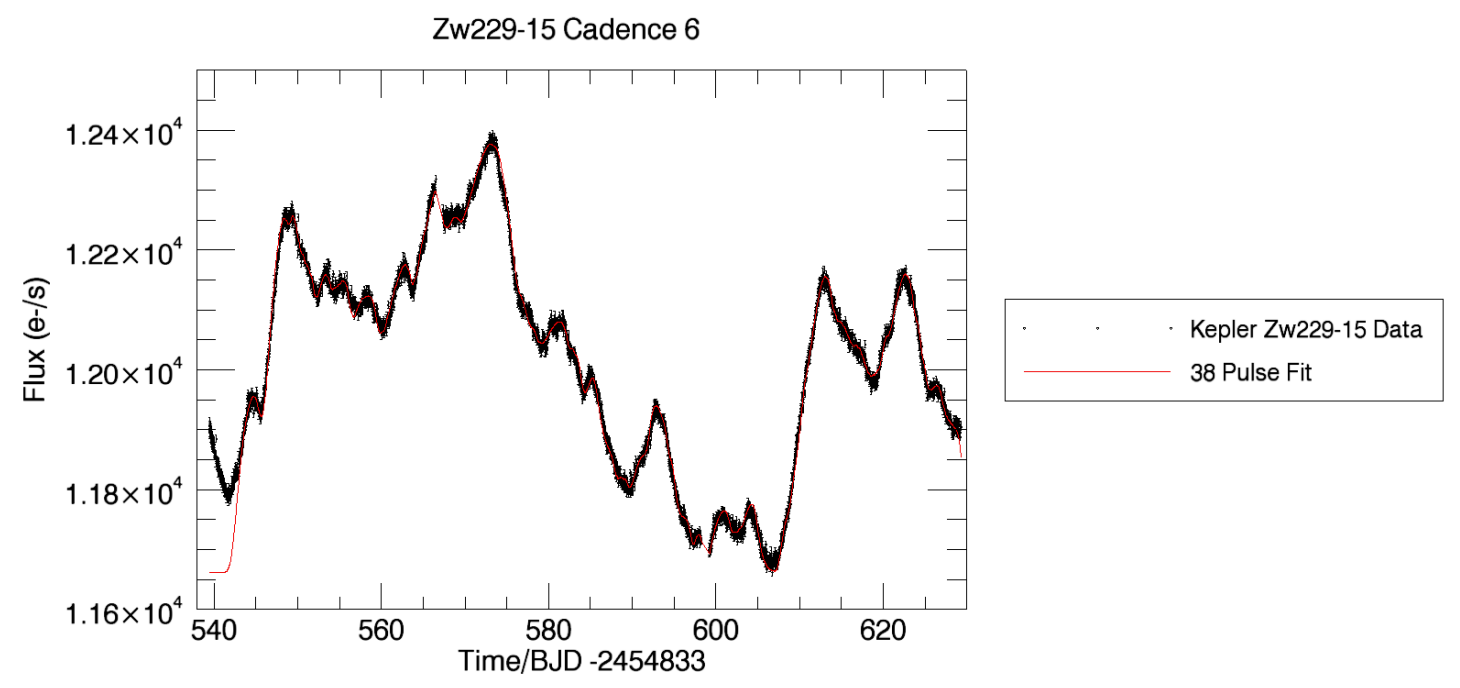

Fig. 18. - Zw 229-15 cadence 6 fitted light curve, where the red lines represent the pulses and the black, data points.

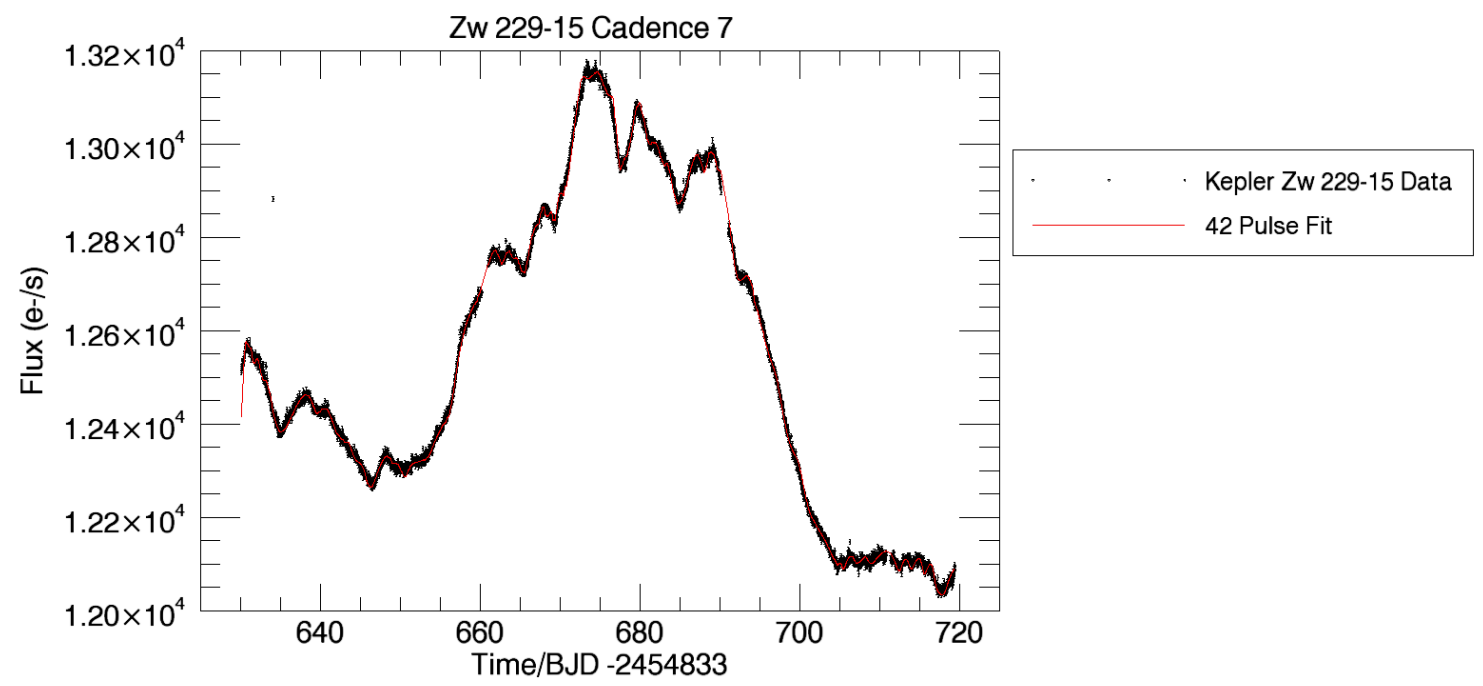

Fig. 19.- Zw 229-15 cadence 7 fitted light curve, where the red lines represent the pulses and the black, data points. 


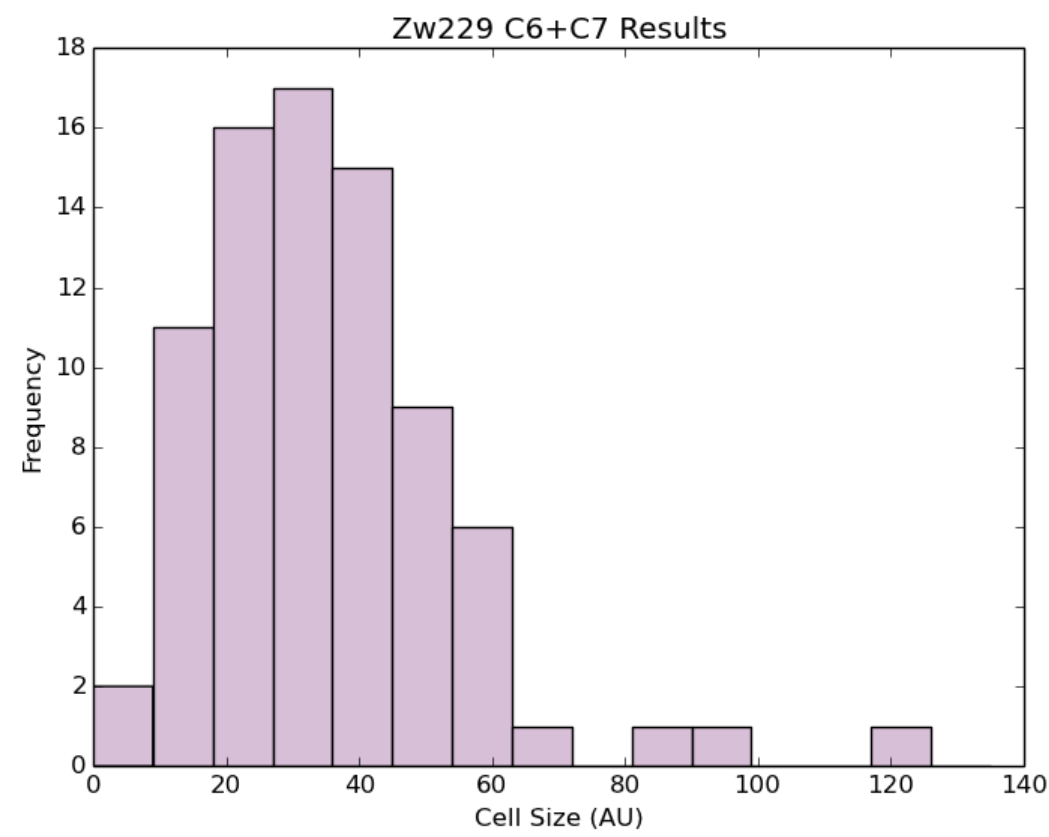

Fig. 20.- Zw 229-15 cadence 6 and 7 frequency distribution of cell size. 


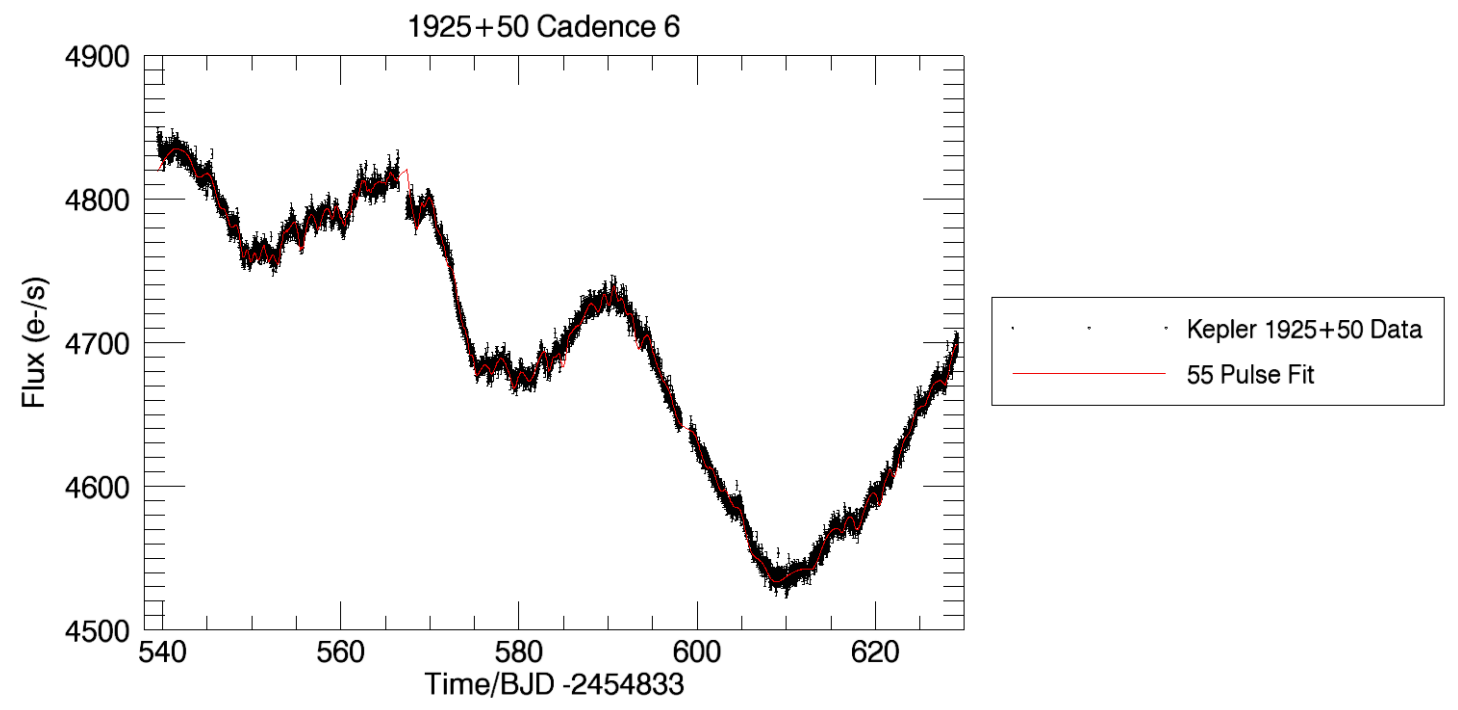

Fig. 21. - KA 1925+50 cadence 6 fitted light curve, where the red lines represent the pulses and the black, data points.

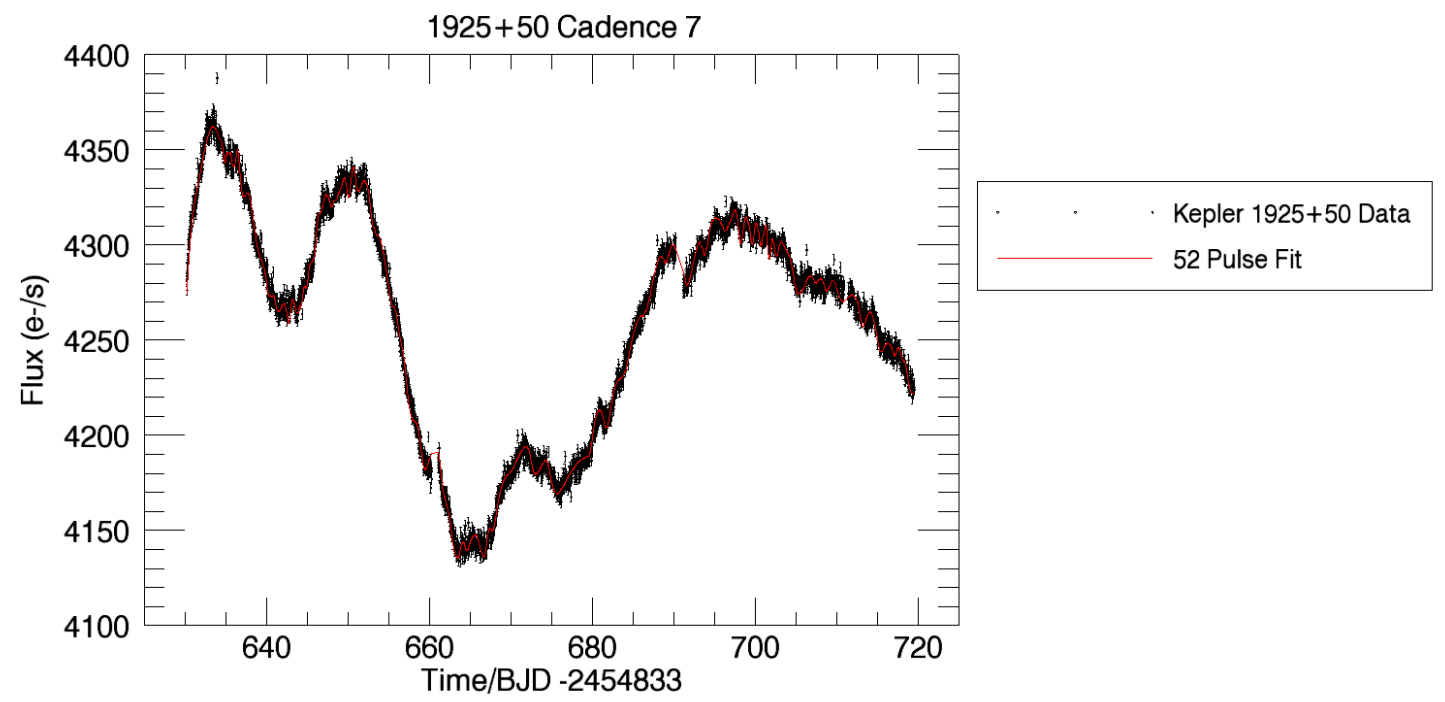

Fig. 22.- KA $1925+50$ cadence 7 fitted light curve, where the red lines represent the pulses and the black, data points. 


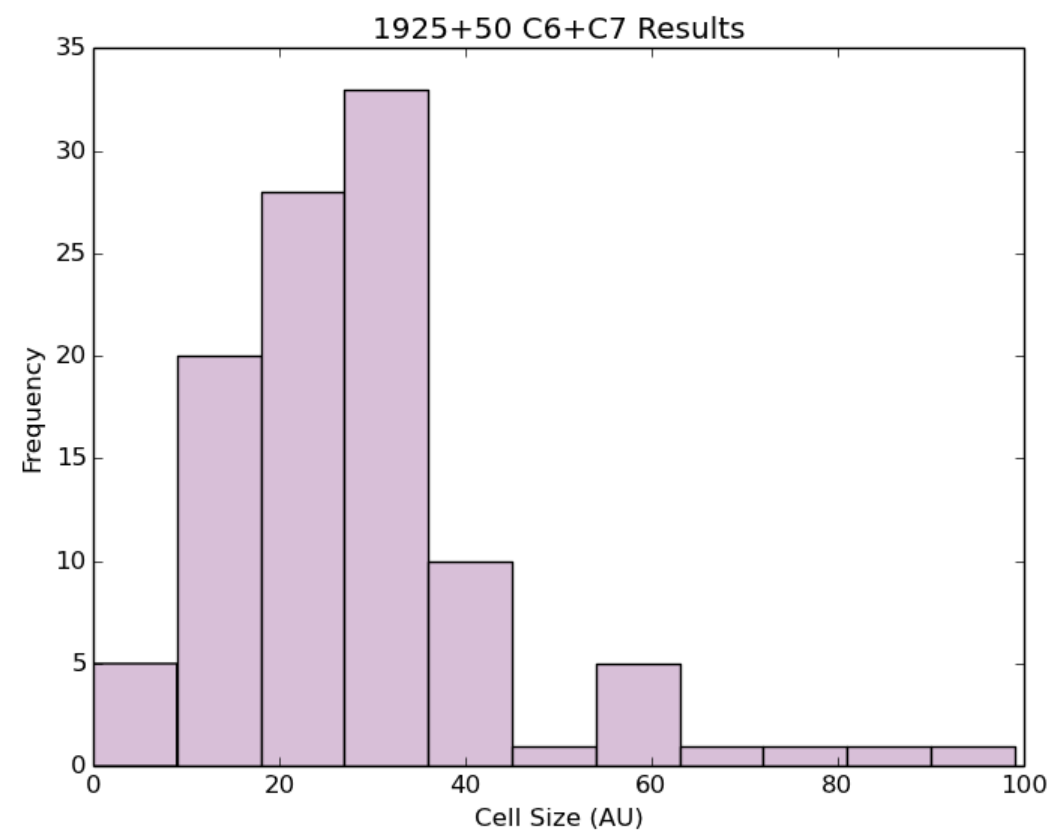

Fig. 23.- KA 1925+50 cadence 6 and 7 frequency distribution of cell size. 


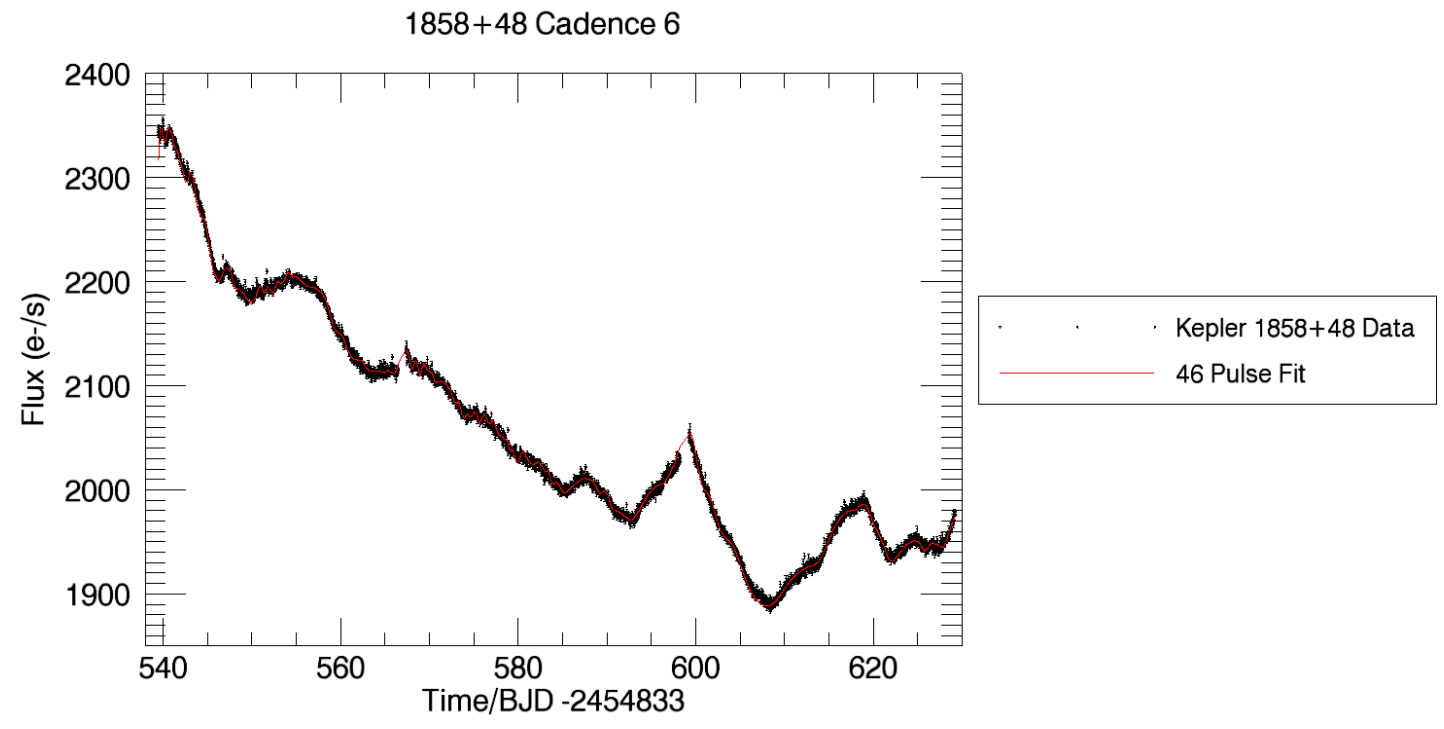

Fig. 24. - KA 1858+48 cadence 6 fitted light curve, where the red lines represent the pulses and the black, data points.

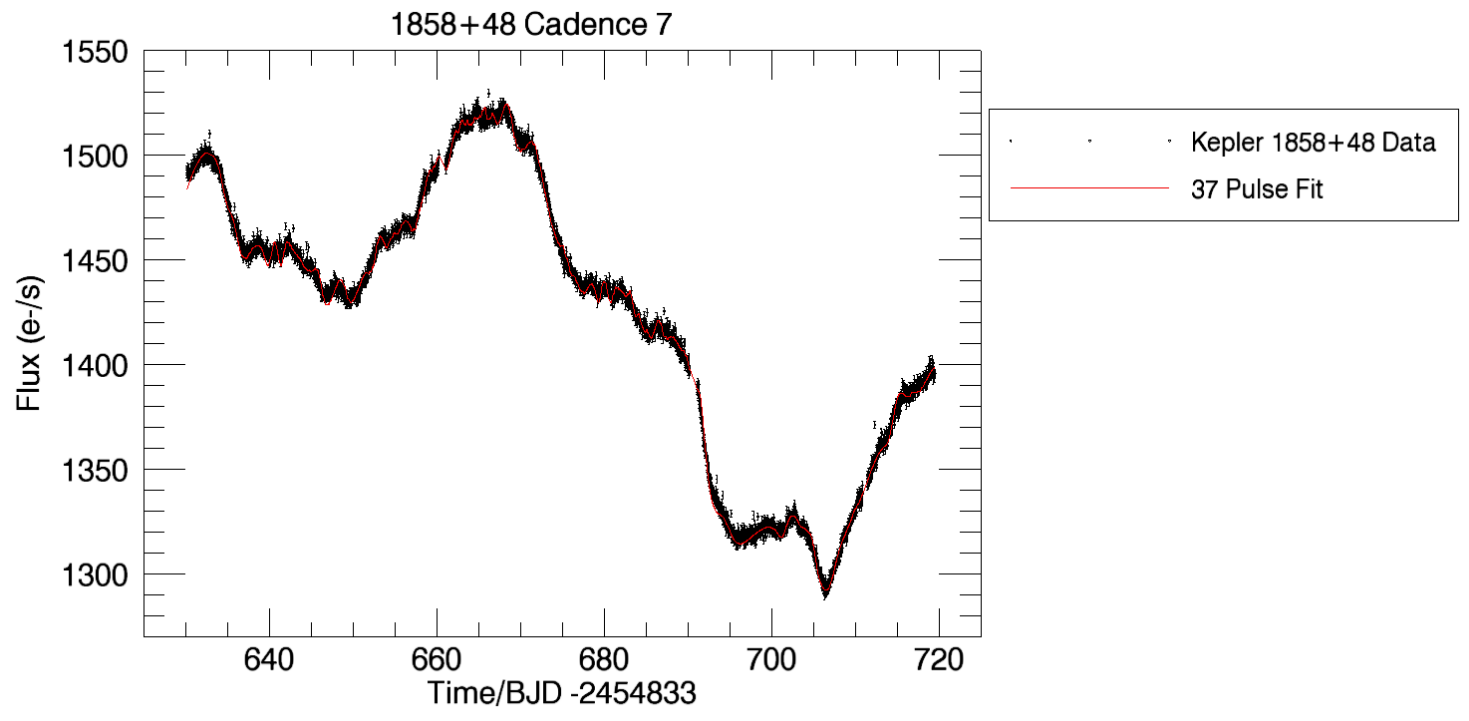

Fig. 25. - KA $1858+48$ cadence 7 fitted light curve, where the red lines represent the pulses and the black, data points. 


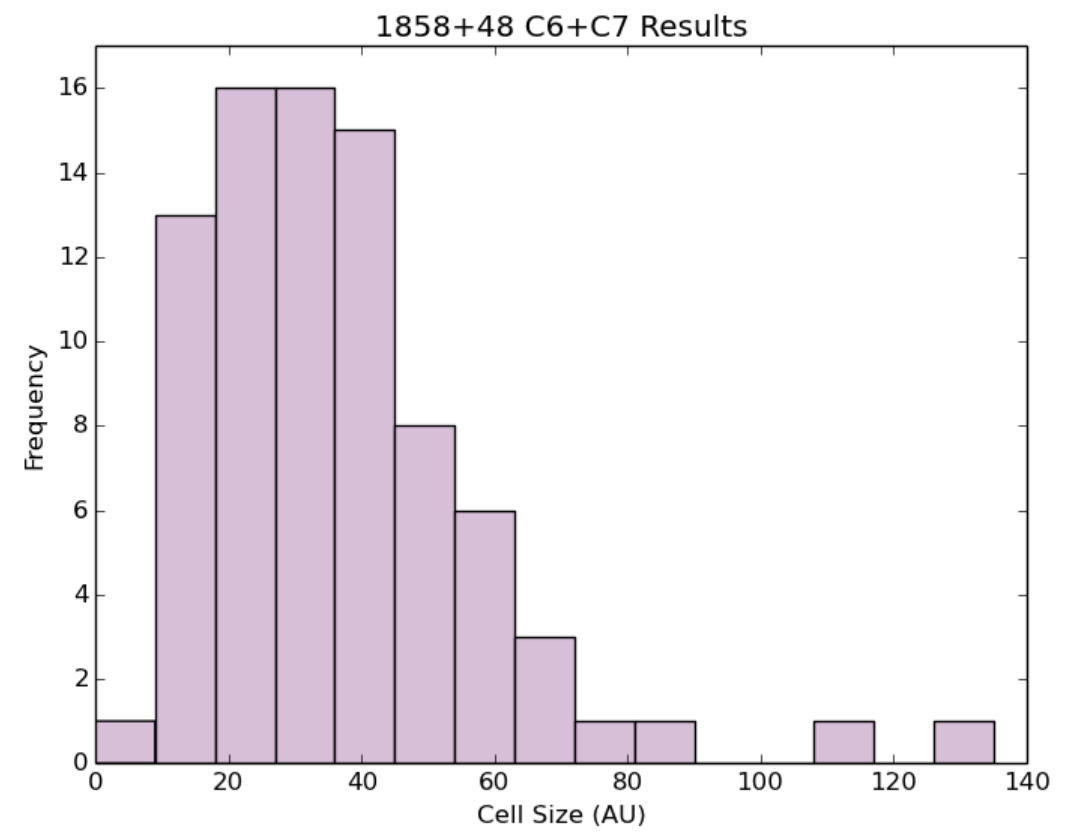

Fig. 26. - KA 1858+48 cadence 6 and 7 frequency distribution of cell size.

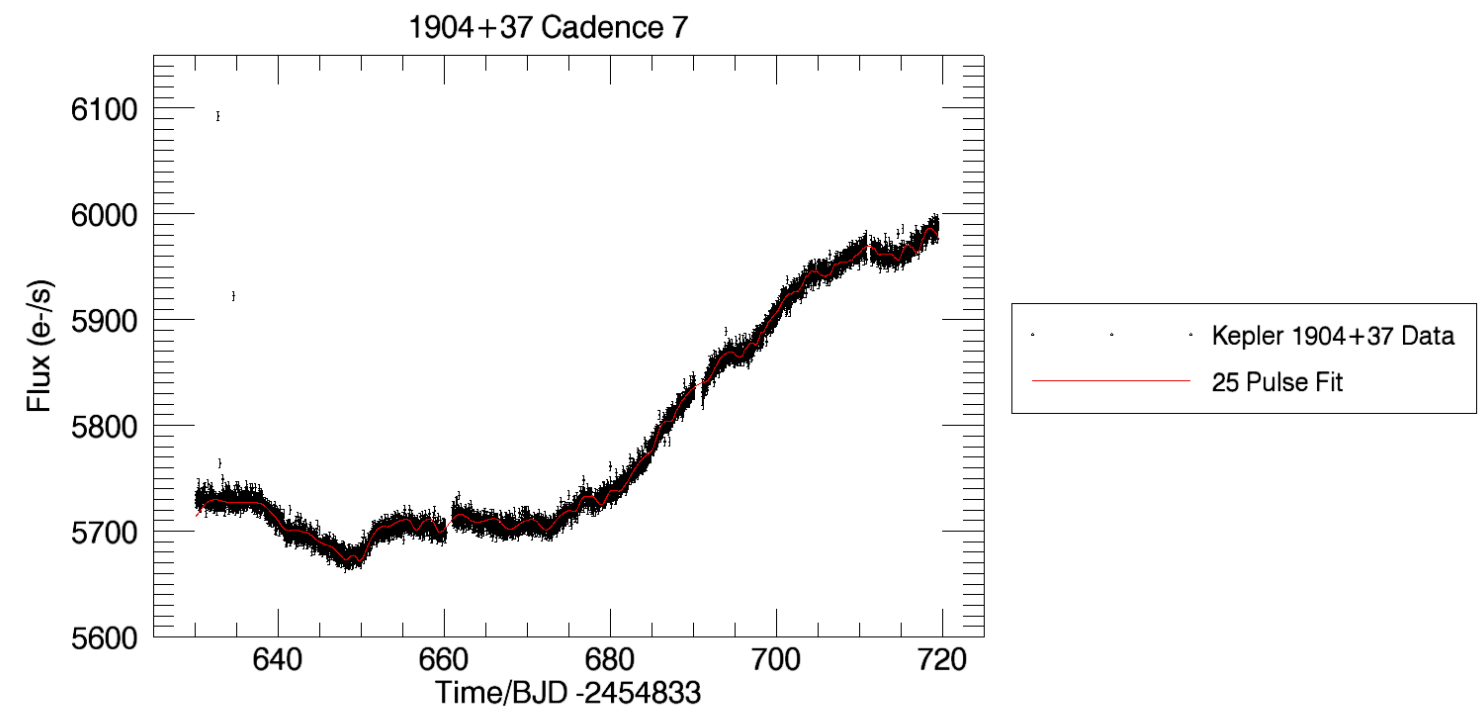

Fig. 27.- KA 1904+37 cadence 7 fitted light curve, where the red lines represent the pulses and the black, data points. 


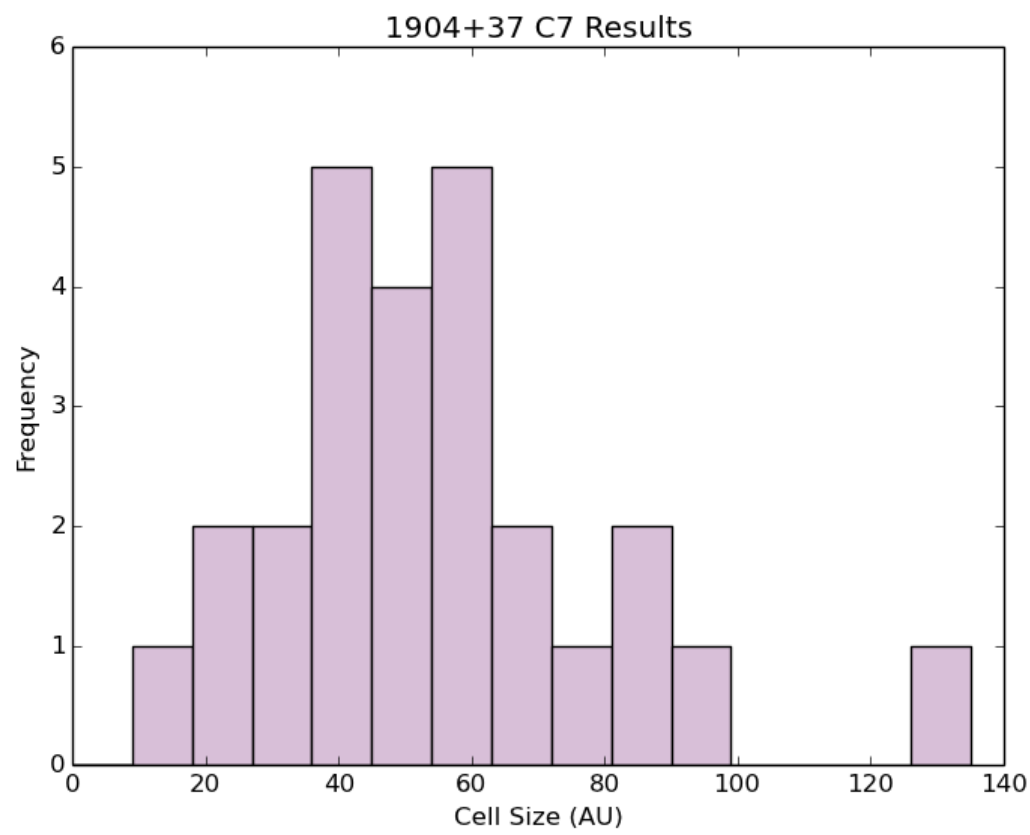

Fig. 28.- KA 1904+37 cadence 7 frequency distribution of cell size. 


\section{Conclusion and Future Work}

The analysis of Kepler objects using the KRM model showed that the average cell size for each object was $\sim 37.4$ AU. The WEBT Campaign produced a 78.88 hour light curve of $0716+78$ fitted with 37 pulses. The average cell size was 36.50 AU. When compared to the light curves in the Kepler study, we see that the average cell sizes are very similar. Because the cell sizes in all objects (including 0716+71) seem to be similar, we can assume that the same processes are occurring in these AGN jets. Furthermore, we can see that there are very few cells that are larger than $60 \mathrm{AU}$, which further agrees with the theory that the larger cells in a turbulent plasma may be unstable and break apart.

In order to verify our results, we would like to further study the Kepler objects using the SARA 0.9 m telescope. Since Kepler does not have standard calibrated magnitudes, we can observe the objects with multiple filters and perform aperture photometry using comparison stars in the field to get a baseline calibration for the Kepler observations. By observing these objects in multiple filters, we can also perform the spectral analysis to determine if there is a difference between the results found in the $0716+71$ spectral study. With regards to the spectral study of $0716+71$, we did not recover the hysteresis loop-like structure as predicted by Kirk, Rieger \& Mastichiadis (1998). Our group is still in the process of replicating the graphs and code that were presented in their paper. It is possible that the $\mathrm{V}$ and I frequency bands are not separated widely enough. Therefore, in order to verify the model, our group plans to write a proposal to use the Keck Observatory's near-infrared and optical telescopes (located in Mauna Kea, Hawaii) to observe 0716+71 simultaneously. Recall, since we only need one isolated pulse to perform the spectral study, we hope that we can recover the spectral hysteresis loop as predicted by Kirk, Rieger \& Mastichiadis (1998).

In addition to the proposed observational runs, we also plan to improve the model to make it more realistic. The current model assumed that all the cells in the jet are 
cylindrical, therefore we need modify the program to allow for spherical cells. In addition, we would like to simulate variability using a distribution of cell sizes consistent with plasma turbulence. Our model also assumes that the magnetic field in the jet is homogenous and does not vary as a function of jet radius. Our model does not consider high energy particle processes and only takes into account the synchrotron emission due to synchrotron radiation and excludes Compton contribution. Furthermore, to make the pulse fitting process more efficient, we plan to automate the fitting process.. 


\section{List of References}

https://archive.stsci.edu/mast faq.php?mission=KEPLER.

http://www.nasa.gov/kepler/keplers-second-light-how-k2-will-work/\#.Uz2Z9vldV0Y.

Allen, L.R., Anderson, B., Conway, R.G., et al. 1962, Mon. Not. Roy. Astron. Soc., 142, 477.

Begelman, M. C., Blandford, R. D., \& Rees, M. J. 1984, Rev. Mod. Phys., 56, 255.

Bhatta G. et al., 2013, A\&A, 558, 92.

Gear W.K., Robson E.I., Brown L.M.J. 1986, Nature, 324, 546.

Ghisellini G., \& Maraschi L. 1989, ApJ, 340, 181.

Jenkins, J. M., Caldwell, D. A., Chandrasekaran, H., et al. 2010, ApJ, 713, L87.

Khembhavi G. \& Narlikar J. 1999, Quasars and Active Galactic Nuclei, Cambridge University Press.

Kirk J.G., Rieger, F.M., \& Mastichiadis, M. 1998, A\&A, 333, 452.

Marscher, A.P., \& Gear, W.K 1985, ApJ, 298, 114.

Marscher, A. 2005, MSAI, 76, 168.

Mc Leod, A., \& Andrew, B.H. 1968, Astrophys. Let., 1, 243.

Meier, D. L., Koide, S., \& Uchida, Y. 2000, Science, 291, 84.

Montagni, F., Maselli, A., Massaro, E., et al. 2006, A\&A, 451, 435.

Mushotzky R. F., Edelson R., Baumgartner W., et al. 2011, ApJ, 743, L12.

Pollock J. T., Webb J. R., \& Azarnia G. 2007, AJ, 133, 487.

Rafle, H., Webb, J.R., \& Bhatta, G. 2012, jSARA, 7, 33. 
Schmidt, M., 1963 Nature 197, 1040.

Sikora M., Begelman M.C., \& Rees M.J. 1994, ApJ, 421, 153.

Sikora, M., Blazejowski, M., Begelman, M. C., et al. 2001, ApJ, 554, 1; Erratum: ApJ 561, 1154 (2001).

Sokolov, A., Marscher, A.P., \& McHardy, I.A. 2004, ApJ, 613, 725.

Takahashi T., Tashiro M., Madejski G., et al. 1996, ApJ, 470, L89.

Torres, D.F., 2004 http://ned.ipac.caltech.edu/level5/March04/Torres/Torres2_ 4.html

Urry, C.M., Padovani, P. 1995, PSAP, 107, 803, http://heasarc.gsfc.nasa.gov/docs/cgro/images/epo/gallery/agns/index.html

Van Cleve, J., Caldwell, D., Thompson, R., et al. 2009, Kepler A Search for Terrestrial Planets, Document KSCI-19033-001 NASA Ames Research Center, Kepler Instrument Handbook Manual (Pasadena, CA: SSC), https://archive.stsci.edu/kepler/manuals/KSCI19033-001.pdf

von Montigny C. et al. 1995, ApJ, 440, 525

Webb, J. R., 2010, BAAS, 21642011W. 14 
APPENDIX I- Pulse Program (Bhatta et al., 2013) Pro Make_pulse, Flare,Tm

COMMON parameters,gama_max,gama_not,cs,us,tacc ; Common parameters shared by the program and the function below

;/ constants/

amp=5.0 ; A normalization number of the amplitude of the pulse

$\mathrm{B}=1.0 \mathrm{e}-3 / \mathrm{amp}$; Maganetic field

$\mathrm{cs}=3.0 \mathrm{e}+8 ;$ Speed of light

us $=0.1 * \mathrm{cs} ;$ Speed of shock

rho_T $=6.65 \mathrm{e}-29$; Thompson scattering constant

$\mathrm{m}_{-} \mathrm{e}=9.1 \mathrm{e}-31$; Mass of electron

mu_0=!pi*4.0e-7 ; Pearmeability for free space

gama_not $=10.0$; Minimum Lorentz factorof the electrons

gama_max $=1000.0$; Maxmimum Lorentz factor of the electrons

beta $\_s=4.0 / 3.0 *$ rho_ $\mathrm{T}\left(\mathrm{m}_{-} \mathrm{e}^{*} \mathrm{cs}\right) *\left(\mathrm{~B} \hat{2} /\left(2 * \mathrm{mu} \_0\right)\right) ; \mathrm{A}$ constant in the KRM equations

tacc $=1 /($ gama_max*beta_s $) ;$ accelaration time

tesc $=2.0 *$ tacc $;$ escape time

$\mathrm{Q}=609.31$; A normalise particle injection rate for the back ground intensity and divided by

2 for the fact that the injection is enhanced by a factor of 2

data_length=gama_max-gama_not ; the difference between the maximum and minimun

Lorentz factors of the electrons

data_interval $=0.5$

data $\_$nums $=$data $\_$length/data $\_$interval 
power $=($ tacc-tesc $) /$ tesc

pwr $=($ tacc + tesc $) /$ tesc

$\mathrm{a}=\mathrm{Q}^{*} \operatorname{tacc}^{*}$ gama_not$\left.\hat{(t a c c / t e s c}\right) *(1$-gama_not/gama_max $) ;$ a constant is equation 2.5

factor $=1 /$ gama $\_$not $-1 /$ gama $\_$max

as $=4.1 \mathrm{e}-36 * \mathrm{~B}$

$\mathrm{Nu}=4.3 \mathrm{e} 14$; Optical frequency for the R filter

time $=50$; Time arrry

$\mathrm{tm}=\mathrm{fltar}($ Time $)$

$\mathrm{t} \_\mathrm{m}=$ fltarr(Time)

Flux =fltarr(Time)

Flux2=fltarr(Time)

Intensity=fltarr(Time)

For $\mathrm{k}=0$, Time- 1 do begin $\operatorname{tm}(\mathrm{k})=(\mathrm{k}+1.0) * \operatorname{tacc} * 0.1$; Time loop

$\mathrm{t}=\mathrm{tm}(\mathrm{k})$

$\mathrm{x} 1=\mathrm{t}^{*} \mathrm{us} /(1-\mathrm{us} / \mathrm{cs}) ;$ Position of shock wave front

gama1=1/(1/gama_max+factor*exp(-t/tacc $)) ;$ Instantaneous Larentz factor given by the equation 2.5

;print,"gamma1 is",gama1

;/This part of the program ensure that incoming gamas are less than

; /gama1

r=gama_not+findgen(data_nums+1.0)*data_interval

;print,r $y 1=$ where(r lt gama1,count) ; Finds the total number of elements statisfying the 
condition

gama=fltarr(count)

gama $=r(0:$ count- 1$)$; Creating subarray from $r$

;print,gama

;/New variables declarations

$\mathrm{N}=$ fltarr(count); particle density

sync _func=ftlarr(count); Green's synchrotron function given by the equation 2.8

$\mathrm{z}=\mathrm{fltarr}$ (count); A term is the equation 2.8

$\mathrm{P}=$ fltarr(count)

$\mathrm{F}=$ fltarr(count); Flux given by the equation 2.11

;/This part of the program calculates the flux

For $\mathrm{i}=0$, count -1 do begin

;Length $=(\mathrm{t}-\mathrm{tb}) /(1-\mathrm{us} / \mathrm{cs})$

$\mathrm{x} 0=$ transcedental $(\operatorname{gama}(\mathrm{i}), \mathrm{t}) * \mathrm{us} * \operatorname{tacc}$; Cooling length calculate by the transecdental function

$\mathrm{N} 1=$ a*gama_max $\hat{2} /(0.9 *$ gama_maxp̂wr*gama(i) 2$) *($ gama_max/gama(i)-t/tacc+x $1 *(1-$

us/cs)/(us*tacc)-1)(tacc/tesc); Number density at the shock wave front

$\mathrm{N} 0=a^{*}$ gama_max $2 /(0.9 *$ gama_maxp̂wr*gama(i) $\hat{2}) *(($ gama_max/gama_not-1)*exp(-

$\mathrm{x} 0)$ )(tacc/tesc) ; Number density at the end of the cooling length

$\mathrm{N}(\mathrm{i})=\mathrm{N} 1-\mathrm{N} 0$

$z(\mathrm{i})=23.8 \mathrm{e}-13 * \mathrm{Nu} / \mathrm{gama}(\mathrm{i}) \hat{2}$

$\mathrm{P}(\mathrm{i})=\mathrm{as} * \mathrm{z}(\mathrm{i}) \hat{0} .3 * \exp (-\mathrm{z}(\mathrm{i}))$; The synchrotron function 
$\mathrm{F}(\mathrm{i})=\mathrm{N}(\mathrm{i}) * \mathrm{P}(\mathrm{i})$

Endfor

Flux $(\mathrm{k})=$ int_tabulated $($ gama,F $) * 4409.98 * 1 \mathrm{e}+23 ; / 4409.98 *$ the first fatctor is correction $\mathrm{tf}=20 \mathrm{j}=\mathrm{k}-\mathrm{tf}$

if $(\mathrm{j}$ lt 1$)$ then Flux2(k)=0 else Flux2(k)=Flux $(k-20)$

norm_factor=int_tabulated $($ gama, $\mathrm{N})$

Endfor

Flare $=2 *($ Flux-Flux 2$)$

Return

End

; This function finds the solution of the trascendental equation ?? for given $\gamma$ and hence estimates the cooling length.

Function transcedental, gama,t ; The inputs for this function are $\gamma$ and time COMMON parameters

$\mathrm{x}=$ findgen $(100000) * 0.001$

$\mathrm{y} 1=($ gama_max/gama_not-1.0)*exp $(-\mathrm{x})$

y2=gama_max/gama-t/tacc $+x *(1-$ us/cs $)-1$

ans $=\min (\operatorname{abs}(y 1-y 2)$,index $)$

return, sol

End 
APPENDIX II- Pulse Fitting Program (Bhatta et al., 2013) Pro Pulse_Fitting

; This program fits the light curve and reproduces it from the given center time, amplitude and width.

; Reading the data

npts $=2613$; Number of points in the file

datain $=$ fltarr $(2$, npts $)$

readf,1,datain

close, 1

; $\mathrm{x}=$ time and $\mathrm{y}=$ flux

$\mathrm{x}=$ datain $\left(0,{ }^{*}\right)$

$\mathrm{y}=$ datain $(1, *)^{*} 1000.0$; Scaling for better numbers

;Read the parameter from the parameter file

openr,2,"C: parameters.dat"

data $=$ fltarr $(4,28)$

readf,2,data

close, 2

center $=$ data $(1, *)$; Second column of the parameters fiel is the center location of the pulse background $=\min (y)$; the minimum of the flux is taken as the background flux level $\mathrm{AMP}=\operatorname{data}(2, *)-$ background

FWHM $=$ data $(3, *) ;$ Fourth column is the width of the pulse Width=FWHM/2.335*13; Scaling to fit the data 
; The flare data obtained from the previous program

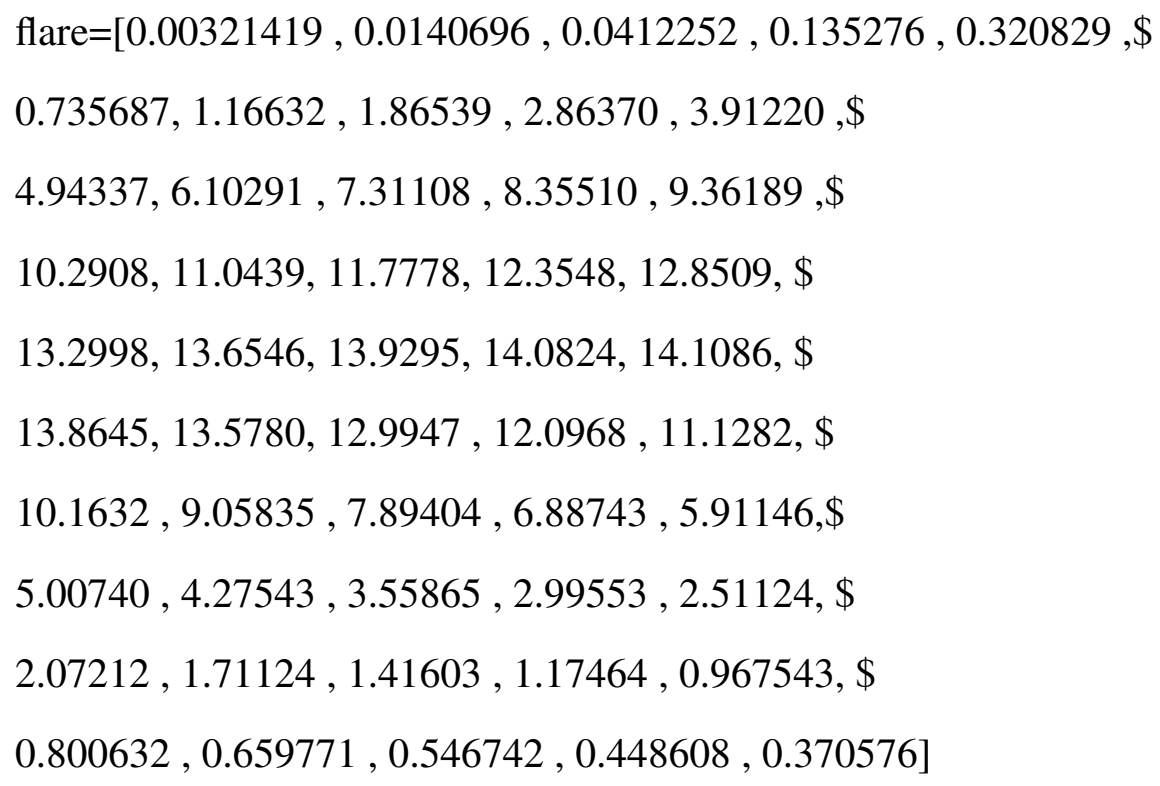

; New variable declartaion

$$
\begin{aligned}
& \text { new_x=fltarr(35,2613) } \\
& \text { flare1=fltarr(35,2613) } \\
& \text { Fitting=fltarr(2613) }
\end{aligned}
$$

For $\mathrm{i}=0,35$ do begin for $\mathrm{j}=0,2612$ do begin new_x $(\mathrm{i}, \mathrm{j})=(\mathrm{x}(\mathrm{j})$-center(i) $) * 50 /$ width(i) +23.0 ; The offset for the center is 23.0 and 50 is the total number of points in the pulse flare $1(\mathrm{i}, \mathrm{j})=$ interpolate $($ flare,new_x $(\mathrm{i}, \mathrm{j})$, cubic=-0.5,missing=0)/14.1086*AMP(i) Endfor 


\section{Endfor}

For $\mathrm{k}=0,2612$ do begin

Fitting $(\mathrm{k})=\operatorname{total}\left(\right.$ flare $\left.1\left({ }^{*}, \mathrm{k}\right)\right)+\min (\mathrm{y})$; The pusles co-adding on top of the background Endfor

plot,x,y ; Plotting the light curve

oplot,x,fitting,psym=3; Overplotting the fitting with different symbol

print,correlate(y,flare1); Correlation coefficient

End 
APPENDIX III- Pulse Fitting Data

Table 4:: Zw 229-15 Pulse Fitting Data- Cadence 6

\begin{tabular}{|c|c|c|}
\hline Pulse Number & Location & Size (AU) \\
\hline 1 & 544.5 & 45.31 \\
\hline 2 & 548.2 & 45.81 \\
\hline 3 & 550.35 & 27.02 \\
\hline 4 & 551.5 & 13.18 \\
\hline 5 & 553.15 & 49.43 \\
\hline 6 & 555.5 & 29.66 \\
\hline 7 & 556.65 & 40.86 \\
\hline 8 & 558.55 & 42.84 \\
\hline 9 & 559.65 & 44.49 \\
\hline 10 & 562.7 & 54.54 \\
\hline 11 & 565.9 & 39.87 \\
\hline 12 & 568.15 & 32.95 \\
\hline 13 & 572.5 & 125.9 \\
\hline 14 & 577.85 & 31.31 \\
\hline 15 & 581.38 & 62.62 \\
\hline 16 & 583.3 & 16.47 \\
\hline 17 & 584.9 & 32.95 \\
\hline 18 & 587 & 49.43 \\
\hline 19 & 589 & 16.47 \\
\hline 20 & 591.2 & 39.54 \\
\hline 21 & 593.15 & 26.36 \\
\hline 22 & 594.55 & 19.77 \\
\hline
\end{tabular}

Continued on next page 
Table 4 - Zw 229-15 Pulse Fitting Data-Cadence 6

\begin{tabular}{|c|c|c|}
\hline Pulse Number & Location & Size (AU) \\
\hline 23 & 596.2 & 29.66 \\
24 & 598 & 13.18 \\
25 & 600.8 & 36.25 \\
26 & 602.6 & 13.18 \\
27 & 604.15 & 28.01 \\
28 & 608.8 & 26.36 \\
29 & 610.1 & 23.07 \\
30 & 612.8 & 59.32 \\
31 & 616.2 & 39.54 \\
32 & 618.7 & 39.54 \\
33 & 620.2 & 11.53 \\
34 & 622.4 & 59.32 \\
35 & 624 & 16.47 \\
36 & 624.7 & 16.47 \\
37 & 626.5 & 32.95 \\
38 & 628.7 & 29.66 \\
\hline
\end{tabular}

Table 5:: Zw 229-15 Pulse Fitting Data- Cadence 7

\begin{tabular}{|c|c|c|}
\hline Pulse Number & Location & Size (AU) \\
\hline 1 & 630.5 & 18.12 \\
2 & 631.3 & 8.23 \\
3 & 631.9 & 18.12 \\
\hline
\end{tabular}

Continued on next page 
Table 5 - Zw 229-15 Pulse Fitting Data-Cadence 7

\begin{tabular}{|c|c|c|}
\hline Pulse Number & Location & Size (AU) \\
\hline 4 & 632.65 & 47.78 \\
\hline 5 & 637.85 & 88.98 \\
\hline 6 & 641.8 & 41.19 \\
\hline 7 & 643.27 & 22.41 \\
\hline 8 & 645 & 42.84 \\
\hline 9 & 648.3 & 42.84 \\
\hline 10 & 650.6 & 29.66 \\
\hline 11 & 652.6 & 32.95 \\
\hline 12 & 655.05 & 36.25 \\
\hline 13 & 658.2 & 47.78 \\
\hline 14 & 661.8 & 54.38 \\
\hline 15 & 664.65 & 36.25 \\
\hline 16 & 665 & 16.47 \\
\hline 17 & 667.1 & 34.93 \\
\hline 18 & 668.7 & 23.07 \\
\hline 19 & 669.85 & 29.66 \\
\hline 20 & 670.37 & 22.41 \\
\hline 21 & 672.15 & 29.66 \\
\hline 22 & 674.2 & 31.31 \\
\hline 23 & 676.3 & 31.31 \\
\hline 24 & 677.7 & 21.42 \\
\hline 25 & 679.53 & 47.78 \\
\hline 26 & 682.1 & 18.127 \\
\hline 27 & 682.8 & 47.13 \\
\hline
\end{tabular}

Continued on next page 
Table 5 - Zw 229-15 Pulse Fitting Data-Cadence 7

\begin{tabular}{|c|c|c|}
\hline Pulse Number & Location & Size (AU) \\
\hline 28 & 686.95 & 68.22 \\
39 & 689.97 & 35.26 \\
31 & 691.3 & 19.77 \\
32 & 693.3 & 61.63 \\
33 & 696.7 & 42.84 \\
34 & 700.9 & 36.25 \\
35 & 705.15 & 98.87 \\
36 & 706.5 & 6.59 \\
37 & 708 & 19.77 \\
38 & 710.7 & 19.77 \\
39 & 713.4 & 49.43 \\
40 & 714.9 & 16.47 \\
41 & 716.4 & 19.77 \\
42 & 719.5 & 14.83 \\
\hline
\end{tabular}

Table 6:: KA 1858+48 Pulse Fitting Data- Cadence 6

\begin{tabular}{|c|c|c|}
\hline Pulse Number & Location & Size (AU) \\
\hline 1 & 539.5 & 14.50 \\
2 & 540.1 & 36.25 \\
3 & 542 & 16.47 \\
4 & 542.49 & 40.86 \\
\hline
\end{tabular}

Continued on next page 
Table 6 - KA 1858+48 Pulse Fitting Data-Cadence 6

\begin{tabular}{|c|c|c|}
\hline Pulse Number & Location & Size $(\mathbf{A U})$ \\
\hline 5 & 544.215 & 32.95 \\
\hline 6 & 546. & 47.58 \\
\hline 7 & 547 & 59.32 \\
\hline 8 & 550.15 & 39.54 \\
\hline 9 & 551.5 & 19.77 \\
\hline 10 & 552.6 & 23.07 \\
\hline 11 & 553.9 & 13.18 \\
\hline 12 & 554.2 & 42.84 \\
\hline 13 & 556.7 & 131.83 \\
\hline 14 & 561.6 & 42.84 \\
\hline 15 & 564.6 & 59.32 \\
\hline 16 & 565.4 & 49.43 \\
\hline 17 & 567.2 & 39.54 \\
\hline 18 & 567.9 & 32.95 \\
\hline 19 & 568.98 & 16.47 \\
\hline 20 & 570.2 & 22.74 \\
\hline 21 & 571.71 & 21.42 \\
\hline 22 & 573.06 & 19.77 \\
\hline 23 & 574.1 & 12.19 \\
\hline 24 & 575 & 32.95 \\
\hline 25 & 576.75 & 21.42 \\
\hline 26 & 578.25 & 24.71 \\
\hline 27 & 579.2 & 13.18 \\
\hline 28 & 580.4 & 26.36 \\
\hline
\end{tabular}

Continued on next page 
Table 6 - KA 1858+48 Pulse Fitting Data-Cadence 6

\begin{tabular}{|c|c|c|}
\hline Pulse Number & Location & Size (AU) \\
\hline 29 & 582.1 & 23.07 \\
\hline 30 & 583.2 & 13.18 \\
\hline 31 & 584.1 & 19.77 \\
\hline 32 & 587 & 115.35 \\
\hline 33 & 589.9 & 13.18 \\
\hline 34 & 592.5 & 46.14 \\
\hline 35 & 595.9 & 54.38 \\
\hline 36 & 598.9 & 42.84 \\
\hline 37 & 599.15 & 28.01 \\
\hline 38 & 601.1 & 36.25 \\
\hline 39 & 603.4 & 59.32 \\
\hline 40 & 612.8 & 79.09 \\
\hline 41 & 617.5 & 65.91 \\
\hline 42 & 619.9 & 32.95 \\
\hline 43 & 621.1 & 16.47 \\
\hline 44 & 624.4 & 65.91 \\
\hline 45 & 627.1 & 23.07 \\
\hline 46 & 629.6 & 39.54 \\
\hline
\end{tabular}

Table 7:: KA 1858+48 Pulse Fitting Data- Cadence 7

\begin{tabular}{|c|c|c|}
\hline Pulse Number & Location & Size (AU) \\
\hline 1 & 631.8 & 164.79 \\
\hline
\end{tabular}

Continued on next page 
Table 7 - KA 1858+48 Pulse Fitting Data-Cadence 7

\begin{tabular}{|c|c|c|}
\hline Pulse Number & Location & Size (AU) \\
\hline 2 & 636.45 & 28.01 \\
\hline 3 & 639.4 & 49.43 \\
\hline 4 & 641.4 & 27.02 \\
\hline 5 & 643.2 & 31.31 \\
\hline 6 & 645.4 & 32.95 \\
\hline 7 & 648.1 & 41.85 \\
\hline 8 & 651.5 & 51.74 \\
\hline 9 & 654.2 & 36.25 \\
\hline 10 & 654.75 & 13.18 \\
\hline 11 & 656.5 & 32.95 \\
\hline 12 & 658.8 & 32.95 \\
\hline 13 & 660.3 & 16.47 \\
\hline 14 & 662.15 & 55.04 \\
\hline 15 & 663.5 & 14.83 \\
\hline 16 & 665.1 & 29.66 \\
\hline 17 & 666.4 & 19.77 \\
\hline 18 & 667.9 & 44.49 \\
\hline 19 & 671.25 & 50.42 \\
\hline 20 & 674.5 & 46.14 \\
\hline 21 & 676.15 & 26.36 \\
\hline 22 & 678.4 & 54.38 \\
\hline 23 & 680.7 & 25.37 \\
\hline 24 & 682.4 & 28.01 \\
\hline 25 & 683.9 & 21.75 \\
\hline
\end{tabular}

Continued on next page 
Table 7 - KA 1858+48 Pulse Fitting Data-Cadence 7

\begin{tabular}{|c|c|c|}
\hline Pulse Number & Location & Size (AU) \\
\hline 26 & 685 & 13.18 \\
27 & 686.3 & 44.49 \\
28 & 689 & 34.60 \\
29 & 691.07 & 31.31 \\
30 & 693.6 & 52.73 \\
31 & 699.3 & 85.69 \\
32 & 702.8 & 28.01 \\
33 & 704.55 & 23.07 \\
34 & 709.2 & 42.84 \\
35 & 713.3 & 69.21 \\
36 & 716.2 & 39.54 \\
37 & 719.4 & 52.73 \\
\hline
\end{tabular}

Table 8:: KA 1925+50 Pulse Fitting Data- Cadence 6

\begin{tabular}{|c|c|c|}
\hline Pulse Number & Location & Size (AU) \\
\hline 1 & 540.7 & 194.45 \\
2 & 546.3 & 44.49 \\
3 & 548.1 & 31.31 \\
4 & 549.2 & 29.66 \\
5 & 550 & 18.12 \\
6 & 551.15 & 22.41 \\
7 & 552.1 & 26.69 \\
\hline
\end{tabular}

Continued on next page 
Table 8 - KA 1925+50 Pulse Fitting Data-Cadence 6

\begin{tabular}{|c|c|c|}
\hline Pulse Number & Location & Size $(\mathbf{A U})$ \\
\hline 8 & 552.96 & 25.37 \\
\hline 9 & 554.8 & 32.62 \\
\hline 10 & 555.3 & 29.66 \\
\hline 11 & 556.9 & 27.35 \\
\hline 12 & 558.68 & 24.88 \\
\hline 13 & 560.1 & 19.77 \\
\hline 14 & 560.58 & 6.59 \\
\hline 15 & 560.78 & 6.59 \\
\hline 16 & 561.2 & 13.18 \\
\hline 17 & 562.3 & 36.25 \\
\hline 18 & 564.7 & 32.95 \\
\hline 19 & 566.7 & 29.66 \\
\hline 20 & 568 & 13.18 \\
\hline 21 & 569 & 6.59 \\
\hline 22 & 569.7 & 56.02 \\
\hline 23 & 572.5 & 32.95 \\
\hline 24 & 572.7 & 13.18 \\
\hline 25 & 573.92 & 18.12 \\
\hline 26 & 574.8 & 6.59 \\
\hline 27 & 576 & 56.02 \\
\hline 28 & 578.9 & 36.25 \\
\hline 29 & 580.9 & 27.02 \\
\hline 30 & 582.8 & 28.67 \\
\hline 31 & 584.3 & 18.12 \\
\hline
\end{tabular}

Continued on next page 
Table 8 - KA 1925+50 Pulse Fitting Data-Cadence 6

\begin{tabular}{|c|c|c|}
\hline Pulse Number & Location & $\operatorname{Size}(\mathbf{A U})$ \\
\hline 32 & 585.4 & 13.18 \\
\hline 33 & 585.8 & 24.71 \\
\hline 34 & 587.85 & 65.91 \\
\hline 35 & 590.3 & 26.36 \\
\hline 36 & 591.2 & 19.44 \\
\hline 37 & 592.45 & 24.71 \\
\hline 38 & 593.85 & 56.02 \\
\hline 39 & 594.5 & 25.70 \\
\hline 40 & 595.5 & 9.88 \\
\hline 41 & 596.7 & 32.95 \\
\hline 42 & 599 & 32.95 \\
\hline 43 & 601 & 29.66 \\
\hline 44 & 602.7 & 26.36 \\
\hline 45 & 604.45 & 29.66 \\
\hline 46 & 606.7 & 39.54 \\
\hline 47 & 611.8 & 62.62 \\
\hline 48 & 615.5 & 44.49 \\
\hline 49 & 617.2 & 16.47 \\
\hline 50 & 619.6 & 41.19 \\
\hline 51 & 621.4 & 16.47 \\
\hline 52 & 623.3 & 33.94 \\
\hline 53 & 625.4 & 29.66 \\
\hline 54 & 627.35 & 29.66 \\
\hline 55 & 629.3 & 27.35 \\
\hline
\end{tabular}


Table 9:: KA 1925+50 Pulse Fitting Data- Cadence 7

\begin{tabular}{|c|c|c|}
\hline Pulse Number & Location & $\operatorname{Size}(\mathbf{A U})$ \\
\hline 1 & 630.65 & 19.77 \\
\hline 2 & 633 & 88.98 \\
\hline 3 & 634.7 & 9.88 \\
\hline 4 & 635.4 & 9.88 \\
\hline 5 & 636.25 & 14.83 \\
\hline 6 & 637.8 & 29.00 \\
\hline 7 & 639.6 & 26.36 \\
\hline 8 & 640.05 & 29.66 \\
\hline 9 & 640.75 & 11.53 \\
\hline 10 & 642.1 & 32.95 \\
\hline 11 & 643.55 & 15.81 \\
\hline 12 & 644.4 & 11.53 \\
\hline 13 & 644.85 & 22.41 \\
\hline 14 & 646.3 & 6.59 \\
\hline 15 & 647.1 & 49.43 \\
\hline 16 & 649.2 & 24.71 \\
\hline 17 & 650.6 & 9.88 \\
\hline 18 & 651.8 & 59.32 \\
\hline 19 & 654.8 & 32.95 \\
\hline 20 & 656.5 & 23.07 \\
\hline 21 & 658.3 & 29.66 \\
\hline 22 & 660.7 & 29.66 \\
\hline
\end{tabular}

Continued on next page 
Table 9 - KA 1925+50 Pulse Fitting Data-Cadence 7

\begin{tabular}{|c|c|c|}
\hline Pulse Number & Location & $\operatorname{Size}(\mathbf{A U})$ \\
\hline 23 & 662.3 & 13.18 \\
\hline 24 & 664.1 & 9.88 \\
\hline 25 & 665.5 & 19.77 \\
\hline 26 & 667.3 & 9.88 \\
\hline 27 & 669.3 & 34.60 \\
\hline 28 & 671.8 & 36.25 \\
\hline 29 & 674.1 & 25.37 \\
\hline 30 & 678.9 & 98.87 \\
\hline 31 & 680.95 & 23.07 \\
\hline 32 & 683.2 & 30.65 \\
\hline 33 & 685.6 & 35.92 \\
\hline 34 & 688.3 & 39.54 \\
\hline 35 & 690.6 & 30.65 \\
\hline 36 & 693.05 & 36.25 \\
\hline 37 & 695.3 & 29.66 \\
\hline 38 & 697.5 & 32.95 \\
\hline 39 & 699.3 & 21.42 \\
\hline 40 & 700.5 & 16.47 \\
\hline 41 & 701.2 & 9.88 \\
\hline 42 & 701.8 & 18.12 \\
\hline 43 & 702.6 & 26.36 \\
\hline 44 & 703.8 & 34.60 \\
\hline 45 & 706.6 & 42.84 \\
\hline 46 & 708.15 & 14.83 \\
\hline
\end{tabular}

Continued on next page 
Table 9 - KA 1925+50 Pulse Fitting Data-Cadence 7

\begin{tabular}{|c|c|c|}
\hline Pulse Number & Location & Size (AU) \\
\hline 47 & 709 & 32.95 \\
48 & 711.7 & 72.50 \\
49 & 714.8 & 29.66 \\
50 & 716.7 & 26.36 \\
51 & 718.1 & 18.78 \\
52 & 719.5 & 23.07 \\
\hline
\end{tabular}

Table 10:: KA 1904+37 Pulse Fitting Data- Cadence 7

\begin{tabular}{|c|c|c|}
\hline Pulse Number & Location & Size (AU) \\
\hline 1 & 630.4 & 82.39 \\
3 & 636.6 & 131.83 \\
4 & 643.1 & 39.54 \\
5 & 646 & 49.43 \\
6 & 649.1 & 16.47 \\
7 & 652.4 & 46.14 \\
8 & 655.5 & 42.84 \\
9 & 658.2 & 29.66 \\
10 & 661.6 & 52.73 \\
11 & 665.9 & 62.62 \\
12 & 670.5 & 56.02 \\
13 & 677.5 & 56.02 \\
\hline
\end{tabular}

Continued on next page 
Table 10 - KA 1904+37 Pulse Fitting Data-Cadence 7

\begin{tabular}{|c|c|c|}
\hline Pulse Number & Location & Size (AU) \\
\hline 14 & 679.6 & 36.25 \\
15 & 684.5 & 98.87 \\
16 & 687.5 & 42.84 \\
17 & 690.9 & 60.97 \\
18 & 694.8 & 56.02 \\
19 & 697.7 & 39.54 \\
20 & 698.2 & 23.07 \\
21 & 701.3 & 82.39 \\
22 & 706.1 & 65.91 \\
23 & 711 & 79.09 \\
24 & 715.8 & 65.91 \\
25 & 716.2 & 26.36 \\
26 & 719.4 & 49.43 \\
\hline
\end{tabular}

